\title{
Zooplankton community structure from tropical temporary ponds during a flood period
}

\author{
Karime de Araujo Paina1,2,* and Maria da Graça Gama Melão \\ ${ }^{1}$ Postgraduate Program in Ecology and Natural Resources (PPG-ERN), Federal University of São Carlos \\ (UFSCar), Rodovia Washington Luís, Km 235, Zip Code 13565-905, São Carlos, SP, Brazil. \\ 2 Department of Hydrobiology, UFSCar, Rodovia Washington Luís, Km 235, Zip Code 13565-905, São Carlos, \\ SP, Brazil. \\ * Corresponding author: karime_paina@hotmail.com
}

Received: 26/03/18 Accepted: $16 / 11 / 18$

\begin{abstract}
Zooplankton community structure from tropical temporary ponds during a flood period

Many ways of measuring ecosystem biodiversity have been used. Besides taxonomic diversity, the functional diversity approach is considered highly predictive of the structure of biological communities and ecosystem processes. Temporary aquatic ecosystems have a great evolutionary and ecological importance, as they are very endemic places with rare species, often endangered by local extinction. The objective of this study was to describe the structure of the zooplankton community of eight Brazilian temporary ponds during the flood period. The organism collections and measures of environmental variables were carried out from $01 / 31$ to $02 / 02 / 2017$. As they are small and ephemeral environments, with very peculiar characteristics, both the taxonomic diversity ( 27 taxa) and the functional one were relatively low, suggesting convergence of the functional characteristics of the species. The zooplankton community was divided into five functional groups and the most significant functional attributes in the grouping of the species were habitat, trophic group and food habit. The functional groups were similar to the taxonomic groupings, except for raptorial carnivorous (Copepoda Cyclopoida and one Rotifera) that comprised the same functional group. In terms of biomass, two species of the Anostraca of the genus Dendrocephalus far exceeded the other groups. It was shown that the presence of these large Branchiopoda has an impact on the composition and numerical density of the zooplankton community, particularly the Cladocera, possibly due to niche overlap.
\end{abstract}

Key words: Dendrocephalus brasiliensis, Dendrocephalus thieryi, Neotropics, biomass, taxonomic diversity, functional diversity, Brazilian savanna, ephemeral ecosystems

\section{RESUMO}

Estrutura da comunidade zooplanctônica em lagoas temporárias tropicais durante um período de cheia

Diversas maneiras de mensurar a biodiversidade dos ecossistemas têm sido utilizadas. Além da diversidade taxonômica, a abordagem da diversidade funcional é considerada altamente preditiva da estrutura das comunidades biológicas e dos processos ecossistêmicos. Ecossistemas aquáticos temporários possuem uma grande importância evolutiva e ecológica, sendo locais de muito endemismo que abrigam espécies raras, muitas vezes ameaçadas de extinção local. O objetivo deste estudo foi descrever a estrutura da comunidade zooplanctônica de oito lagoas temporárias brasileiras durante um periodo de cheia. As coletas dos organismos e medidas de variáveis ambientais foram realizadas de 31/01 a 02/02/2017. Por se tratar de ambientes pequenos e efêmeros, com características muito peculiares, tanto a diversidade taxonômica (27 táxons) quanto a funcional foram relativamente baixas, sugerindo convergência das características funcionais das espécies. A comunidade zooplanctônica foi dividida em cinco grupos funcionais, sendo que os atributos funcionais mais significativos no agrupamento das espécies foram o habitat, o grupo trófico e a hábito alimentar. Os grupos funcionais foram parecidos com os agrupamentos taxonômicos, exceto para os carnívoros raptoriais (Copepoda Cyclopoida e um Rotifera) que compuseram um mesmo grupo funcional. Em termos de biomassa, duas espécies do Anostraca do gênero Dendrocephalus ultrapassaram em muito os outros grupos. Foi 
evidenciado que a presença desses grandes Branchiopoda impacta a composição e a densidade numérica da comunidade zooplanctônica, particularmente dos Cladocera, possivelmente pela sobreposição de nichos.

Palavras chave: Dendrocephalus brasiliensis, Dendrocephalus thieryi, Neotrópicos, biomassa, diversidade taxonômica, diversidade funcional, cerrado, ecossistemas efêmeros

\section{INTRODUCTION}

Temporary aquatic ecosystems are of great evolutionary and ecological importance, having communities that are physiologically adapted and that have developed effective and diversified mechanisms for their colonization, permanence and reproduction. They are very endemic places that harbor rare species, which are often threatened with extinction. Although often neglected, these ecosystems are often "hot spots" of the biodiversity in a region or landscape (Céréghino et al., 2008).

Temporary ponds are very common in arid and semi-arid regions (Brendonck et al., 2008; Atashbar et al., 2014) and are characterized by well defined and predictable periodic dry and flood cycles (Williams, 1996). During floods, characteristic aquatic communities develop (Pérez-Bilbao et al., 2015). Their fauna and flora has physiological, morphological and behavioral adaptations in response to flood oscillations, making these environments strong points of speciation (Williams, 1996). To adapt to temporary conditions, organisms basically develop two strategies: to withstand the dry phase through dormant life stages or to migrate at the beginning of the dry season (Wiggins et al., 1980). In the case of the invertebrate community, predominant in temporary ecosystems, many species guarantee the survival of offspring through diapause eggs. They are r-strategist species, with high reproduction capacity, rapid growth and short life cycles (Williams, 1997).

Despite the importance of temporary aquatic ecosystems and their vulnerability to biodiversity loss, there is little information about them in tropical regions. This lack of information, as well as inadequate management contribute to their deterioration and even disappearance. As they are small, shallow and temporary, they are often considered unproductive areas where disease-transmitting insects live. This, as well as the need for agricul- tural expansion, tend to cause a reduction of these habitats (Perez-Bilbao \& Garrido, 2009; Pérez-Bilbao et al., 2015). The establishment of invasive species, pollution caused by using fertilizers and pesticides, changes in hydrological functioning and climate change are some of the factors that threaten the integrity of this type of ecosystem, which can have an impact on the richness, abundance, dominance and biomass of their communities (Crossetti \& Bicudo, 2005, Pérez-Bilbao et al., 2015).

In Brazil, the semi-arid region of the northeast concentrates the largest number of this type of ecosystems due to the low rainfall and high rate of evaporation. However, environmental and anthropogenic changes, which cause an imbalance in the hydrological regime, contribute to desertification processes, which may lead to their extinction (Maltchik \& Medeiros, 2006). Thus, understanding the functioning of these systems and the patterns of changes in their biological diversity is critical to their preservation as many of them are threatened by human activities or by climate change.

Biological diversity can be measured by taxonomic and functional approaches. The latter considers behavioral, physiological, morphological and life cycle characteristics, which are called functional traits of the species (Violle et al., 2007; Webb et al., 2010; Litchman et al., 2013) and is considered highly predictive of the structure of biological communities and ecosystem processes (Mcgill et al., 2006; Smith et al., 2013).

Although zooplankton plays a major role in aquatic ecosystems as primary consumers, influencing the entire trophic chain, there are no studies about its functional traits in temporary ecosystems in Brazil. Passos $(2012 ; 2017)$ studied the zooplankton community structure of 25 shallow temporary ponds from the same region of this study, but only under the taxonomic approach. Thus, knowing the composition and variations 


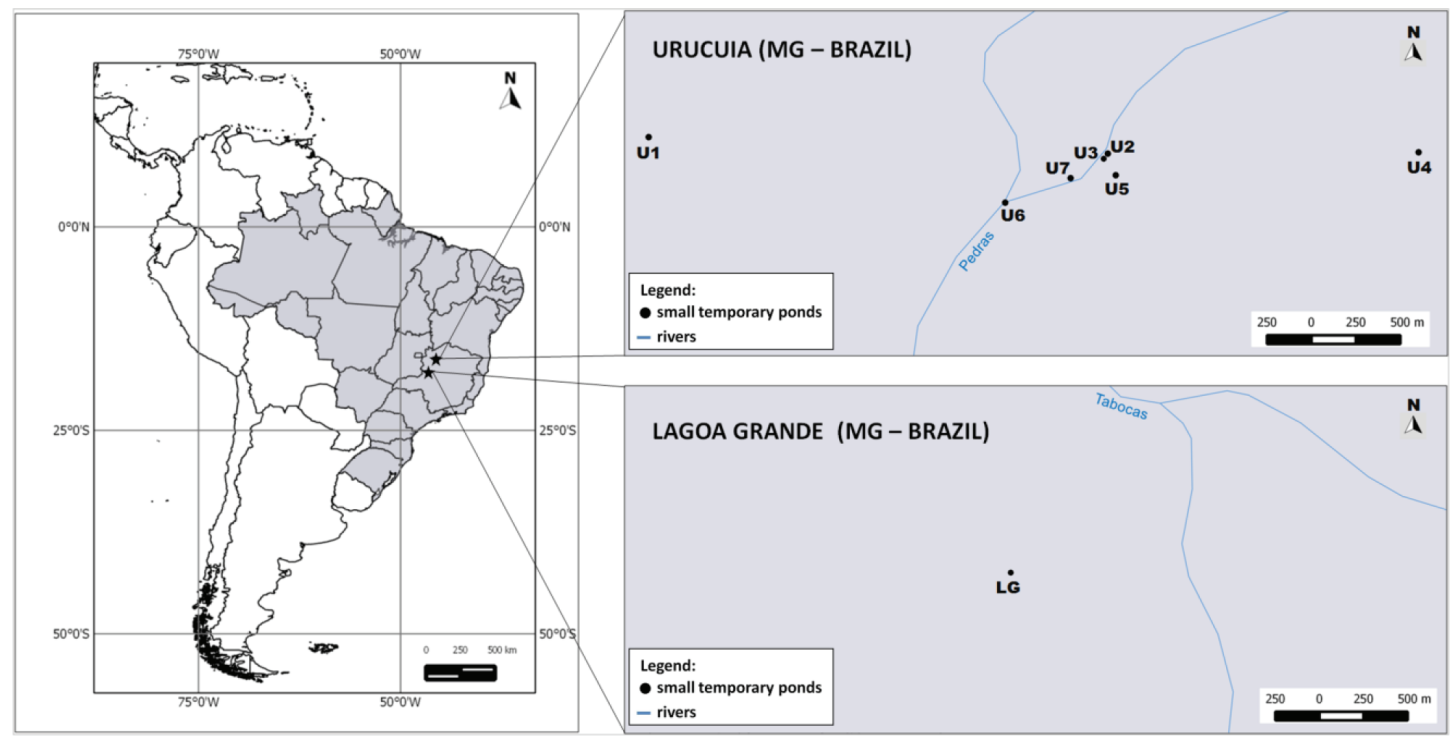

Figure 1. Location map of the study areas, indicating seven temporary ponds in the city of Urucuia (U1 to U7) and one in the city of Lagoa Grande (LG), all in the State of Minas Gerais, Brazil. Mapa de localização das áreas de estudo, indicando sete lagoas temporárias na cidade de Urucuia (U1 a U7) e uma na cidade de Lagoa Grande (LG), todas no Estado de Minas Gerais, Brasil.

in the functional aspects of communities is fundamental in understanding the ecological patterns that shape the different ecosystems (Petchey \& Gaston, 2006). Therefore, the aim of the present study is to describe the zooplankton community structure from tropical temporary aquatic ecosystems during a flood periods, under taxonomic and functional approaches. We can raise the hypothesis that the presence of anostraceans, generalistic supension feeders, as strong competitors, as well as the extreme environmental characteristics of these kind of shallow temporary ecosystems, imply in low richness of zooplanktonic species, specially big filter feeders, and species have strategies to avoid overlap of niches.

\section{METHODS}

\section{Study Area}

Eight temporary ponds were sampled in the state of Minas Gerais (MG), Brazil: seven located in the municipality of Urucuia (U1 to U7) and one in the municipality of Lagoa Grande (LG) (Fig. 1) between January 31 and February 2, 2017. Figure 2 shows an overview of the studied environments and their geographic coordinates. These ponds usually flood between November and March, which is the period of greater precipitation in the northwest of MG. In this period, rainfall is usually around $600 \mathrm{~mm}$, according to the average of the three years before the sampling period of this study (Embrapa, 2013; Simge, 2015). Temporary ponds from this region are very shallow, even with maximum rainfall, rarely exceeding $1.0 \mathrm{~m}$ of profundity (Passos 2012; 2017).

To characterize the sampled ecosystems, the following limnological variables were measured: depth and transparency of the water column (Secchi disk), $\mathrm{pH}$ ( $\mathrm{pH}$ meter Analion PM608), conductivity (conductivity analyzer C708), concentrations of total nitrogen - N (Valderrama, 1981), total phosphorus - P (Mackereth, 1978), suspended solids (total, organic and inorganic) (Cole, 1975) and chlorophyll a (Golterman et al., 1978). Samples were taken in triplicate at one point in the middle of each pond.

\section{Zooplanktonic Community}

Zooplankton was collected using a $68 \mu \mathrm{m}$-mesh plankton net (20 cm-diameter) in open and 
central areas of the ponds. Only in the LG pond, which was very shallow, was water collected using a bucket $(20 \mathrm{~L})$ and then concentrated through the plankton net. The volume of filtered water in each sample was approximately $100 \mathrm{~L}$ and was calculated based on the distance covered by the net and its radius (cylinder volume formula). Collection procedures were performed 3 times in each pond and samples were then fixed in $4 \%$ formaldehyde. Specimens at species level

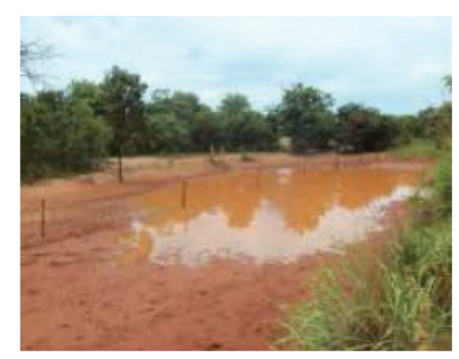

U1 - 160' $54.4^{\prime \prime} \mathrm{S} 45^{\circ} 37^{\prime} 06.5^{\prime \prime} \mathrm{W}$

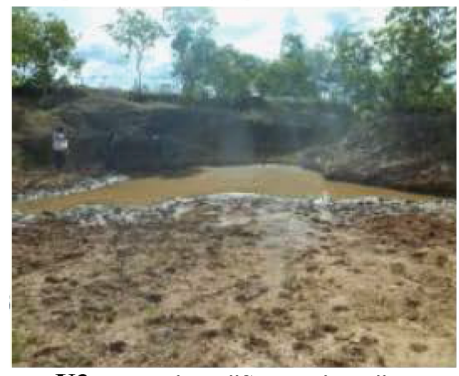

U3 - 16 $07^{\circ} 02.3^{\prime \prime} \mathrm{S} 45^{\circ} 34^{\prime} 14.5^{\prime \prime} \mathrm{W}$

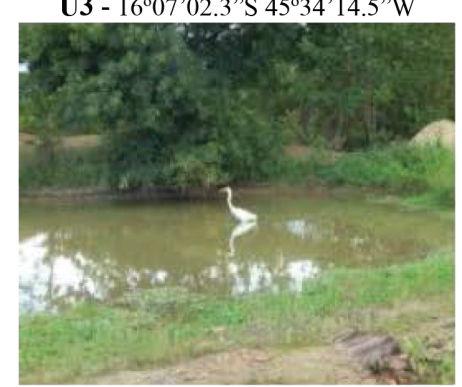

U5 - 1607'10.4”S 45³4'11.5”W

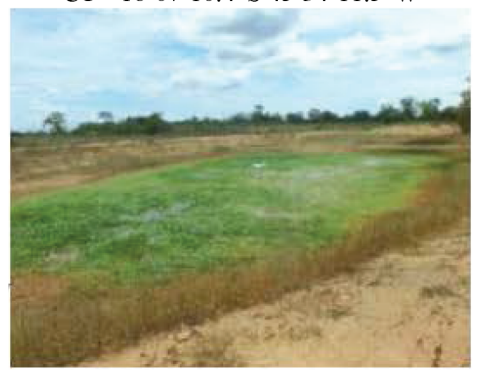

U7 - 16 $6^{\circ} 07^{\prime} 11.5^{\prime \prime} \mathrm{S} 45^{\circ} 34^{\prime} 28.6^{\prime \prime} \mathrm{W}$

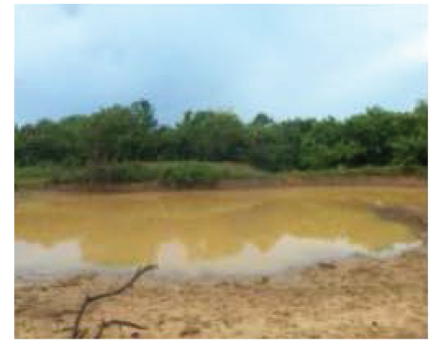

U2 - $16^{\circ} 07^{\prime} 02.3$ 'S $45^{\circ} 34^{\prime} 14.5^{\prime \prime} \mathrm{W}$

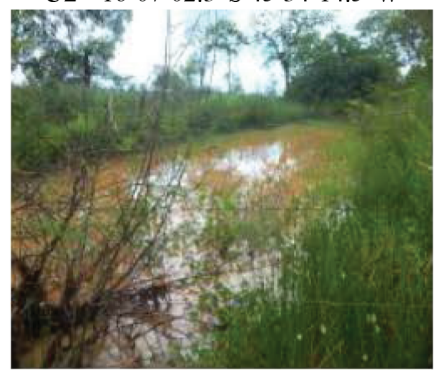

U4 - 16 $6^{\circ} 07^{\prime} 01.8^{\prime \prime} \mathrm{S} 45^{\circ} 32^{\prime} 16.8^{\prime \prime} \mathrm{W}$

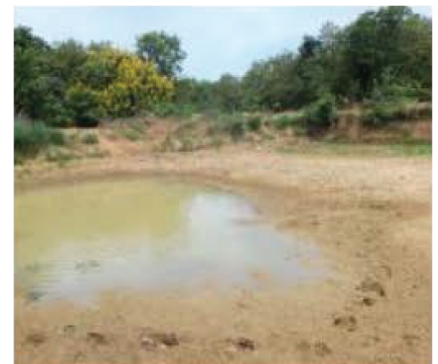

U6 - 1607'32.4”S 45³4'29.6”W

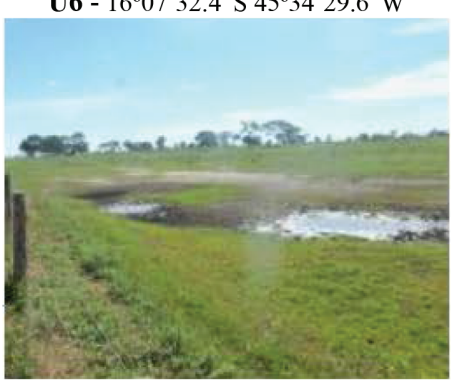

LG - 17²48'01.1”S 463' 41.8”W

Figure 2. Overview of the studied temporary ponds, with the designation codes and geographical coordinates of each one. Ponds denominated U1 to U7 are located in the city of Urucuia, while the pond denominated LG is located in the city of Lagoa Grande, all in the State of Minas Gerais, Brazil. Visão geral das lagoas temporárias estudadas, acompanhadas dos códigos de denominação e coordenadas geográficas de cada uma. As lagoas denominadas de U1 a U7 são localizadas na cidade de Urucuia, enquanto que a lagoa denominada LG está localizada na cidade de Lagoa Grande, todas no Estado de Minas Gerais, Brasil. 
were identified using a specialized bibliography. For qualitative and quantitative analyses, a stereoscopic microscope Leica ${ }^{\circledR}$ model MZ6 with magnification of up to 50 times and a Zeiss ${ }^{\circledR}$ optical microscope were used with a magnification up to 1000 times, both with a micrometered eyepiece. To estimate the relative abundances (population density expressed in individuals $\mathrm{m}^{-3}$ ), individuals of sub-samples were counted in a Sedgewick-Rafter chamber (Rotifera and protozoans), under an optical microscope using grid acrylic plaques (Anostraca, Cladocera and Copepoda), under a stereoscopic microscope. Sub-samples were counted until no statistical variation was observed or no new species were found. In addition, the remaining material was examined at a smaller magnification to search for larger and less abundant taxa.

The length was also measured for each species and the biomass of crustaceans and rotifers were calculated from published regression equations. For rotifers, biomass was estimated from the biovolume calculated by means of specific mathematical formulas corresponding to the geometric forms that most resemble individuals (Ruttner-Kolisko, 1977). Thirty individuals of each species were measured under an optical microscope or, for those species that did not have this number; all individuals in the sample were measured. The biovolume was converted to wet weight considering the specific density of $1.0\left(1 \mu \mathrm{m}^{3}=\right.$ $10^{-6} \mu \mathrm{g}$ wet weight) and then the correction factor 0.1 was used to convert wet weight to dry weight (Bottrell et al., 1976). For Cladocera and Copepoda, biomass was determined by linear regression relating body length - $\mathbf{L}(\mathrm{mm})$ and weight $\mathbf{W}(\mu \mathrm{g})$, that is, $\mathbf{L n} \mathbf{W}=\mathbf{L n} \mathbf{a}+\mathbf{b} \mathbf{L n} \mathbf{L}$, where Ln a is 1.7512 for Cladocera and 1.9526 for Copepoda and $\mathbf{b}$ is 2.6530 for Cladocera and 2.3990 for Copepoda (Bottrell et al., 1976).

\section{Functional Diversity}

Based on the literature (Barnett et al., 2007, 2013; Sodré, 2014; Silva, 2015), eight functional traits were selected for all zooplanktonic species present in the studied ecosystems: (1) body length; (2) trophic group (herbivores, carnivores, omnivores, omnivores/carnivores and omni- vores/herbivores); (3) feeding type (scrapers, suspension feeders, raptorial, sucking, aspiration and pseudopods); (4) type of reproduction (sexual or asexual); (5) food size range; (6) habitat (pelagic ou littoral); (7) number of reproductions per flood period (one or more); (8) duration of embryonic development.

Regarding the trophic group, we consider the predominant feeding of each species, although in many of them there may be some flexibility in the type of food ingested, for example, some herbivores can also feed on bacteria; we considered omnivorous species that do not have a restricted diet, having two or more types of food preference. Based on Hopp \& Maier (2005), for Copepoda Cyclopoida, we consider the groups omnivores/carnivores and omnivores/herbivores in order to contemplate young (omnivores) and adults (carnivores or herbivores)

Concerning the feeding type, scrapers were considered those that capture particles associated with any type of substrate; suspension feeders generate water flow in the search for food, capturing particles that are suspended (active search and passive capture); raptorial are the predators (both searching and capturing food are active); suckers are those who catch and suck on algae or small animals by means of an eversible mastax; aspiration to those that catch the prey due to aspiration created by sudden mastax dilatation; and those that use pseudopods to capture food (amoeboid protozoa). For the type of reproduction, we considered sexual or asexual.

In order to establish the size ranges of the food particles, we used the planktonic group sizes presented by Kalff (2002). For this study, we established five food size ranges: $0.2-10 \mu \mathrm{m}$; $10-15 \mu \mathrm{m} ; 15-40 \mu \mathrm{m} ; 40-100 \mu \mathrm{m} ; 60-300 \mu \mathrm{m}$. We considered the predominant feeding interval for each species as animal feed is not restricted to the established size classes. Femtoplankton (virus and bacteria $<0.2 \mu \mathrm{m}$ ) and nanoplankton (the smallest phytoplankton, $\sim 0.5$ to $2 \mu \mathrm{m}$ ) are food sources for Protozoa and Rotifera, although they may also be part of the feeding of filtering crustaceans such as daphnids. Nanoplankton (from 2 to $30 \mu \mathrm{m}$; often flagellated phytoplankton) is the main food source for micro and macrozooplankton, and small microplankton ( 30 to $70 \mu \mathrm{m}$ ) food 
for macrozooplankton. Microplankton $(>70 \mu \mathrm{m})$ and mesoplankton $(200-20000 \mu \mathrm{m}$; large cells and colonies) are food sources for macrozooplankton and pelagic or benthic omnivores, as well as protozoa and animals of the micro and macrozooplankton. Various studies have provided us with a basis for classifying animals in different food ranges (Burn, 1968; Pourriot, 1977; Ferguson et al., 1982; Schoenberg \& Carlson, 1984; Infante \& Edmondson 1985; Knoechel \& Holtby, 1986; Bodgan and Gilbert, 1987; Chaoruangrit et al., 2017; Bern, 1994; DeMott, 1995; Pagano, 2008; Hopp \& Maier, 2005).

Regarding the habitat, although the studied ponds were very small and shallow, which makes it difficult to separate the species, the classification took into consideration the species that usually live in open waters (pelagic) or those that usually associate with some type of substrate, such as macrophytes, filamentous algae or sediments (littoral), according to the literature (Barnett et al., 2007; 2013).

For the duration of embryonic development (tED), we considered mean values found in the literature $\left(25\right.$ to $\left.27^{\circ} \mathrm{C}\right)$ for the same species from this study or as close as possible. Although protozoa do not have an embryonic development, we include them in the lowest category, since the time it takes to generate a new individual by bipartition is very short. We obtained the tED data from the following literature: Hutchinson 1957; Edmondson, 1960, 1965, 1974; Gras \& St-Jean, 1969, 1976, 1983; Edmondson \& Winberg, 1971; Herzig, 1983; Yúfera, 1987; Vijverberg, 1989; Smirnov, 1992; Hardy \& Duncan, 1994; Wyngaard et al., 1994; Ritzler, 1995; Irvine \& Waya, 1999; Kepeler, 1999; Melão, 1997, 1999; Maia-Barbosa, 2000; Dumont \& Negrea, 2002; Kalff 2002; Hausmann et al., 2003; Melão \& Rocha, 2004; Choueri et al., 2007; Santos-Wisniewski \& Rocha, 2007; Ma et al., 2010; Brito et al., 2016.

\section{Data analysis}

Qualitative and quantitative analysis of zooplankton were made according to CETESB (2000) and Bicudo \& Bicudo (2004). In order to investigate the correlations between environmental variables and the numerical density of zooplankton community of the eight temporary ponds studied, a canonical correspondence analysis (CCA) was performed, by means of the software free R 3.4.2 (R Development Core Team, 2017), and a Pearson correlation coefficient analysis was also performed, where the standard correlation coefficients between the scores of the samples derived from the species density data and the scores of the samples obtained from linear combinations of the environmental variables are presented The Pearson correlation coefficient measures the degree of correlation between two metric scale variables, as well as the direction of the same; if it is a positive correlation (directly proportional $=+1$ ), negative (inversely proportional $=-1$ ) or null (absence of correlation, $=0$ ). The frequency of occurrence (\%) of each species was calculated considering data from all ponds by the formula $\mathrm{F}=100 * \mathrm{~Pa} /$ $\mathrm{P}(\mathrm{Pa}=$ number of samples with a given species; $\mathrm{P}=$ total number of samples) (Dajoz, 1983).

Based on the data from the functional traits of each species, the definition of functional groups was performed by multivariate cluster analysis (Pla et al., 2012). A dissimilarity analysis was performed using the Euclidean method to generate a dendrogram, using the Ward method, which produces better-defined groupings than other methods As the clustering algorithms do not determine an ideal number of groups, from the dendrogram it was possible to observe changes in the levels of dissimilarity for the successive stages of the clustering. The straight lines of the pooled individuals are of height corresponding to the level at which the individuals are considered similar. The software used was the free R 3.4.2 (R Development Core Team, 2017), using the FD package (Laliberté and Legendre, 2010; Laliberté et al., 2014). In order to verify the existence of a correlation between the environmental parameters and the functional groups, canonical correspondence analysis (CCA) was performed using the free software R 3.4.2 (R Development Core Team, 2017). The level of significance between the environmental and biotic parameters was also analyzed using the Monte Carlo test, establishing a $p \leq 0.5$.

The functional dispersion index (FDis) was calculated, which ranges from 0 to 1.0 , and 
reflects how the species are distributed in the environment, also relating the number of functional traits, the number of species and their respective abundances (Cianciaruso, 2009; Sodré, 2014; Silva, 2015). This index was calculated from two matrices: species $\mathrm{x}$ functional traits and species $x$ relative abundances using the FD package of the free software R 3.4.2.

To analyze taxonomic diversity, Shannon Diversity $\left(\mathrm{H}^{\prime}\right)$ index were calculated through the Vegan package of free software R 3.4.2.

\section{RESULTS}

Limnological variables measurements recorded in the studied temporary ponds (Table 1) indicate that, in general, water transparency was very low; $\mathrm{pH}$ was slightly acid to neutral; there was low conductivity; the concentrations of total nutrients ( $\mathrm{N}$ and $\mathrm{P}$ ) were very high; the concentrations of suspended solids were variable (the largest portion was inorganic); and chlorophyll $a$ concentrations were very low.

The zooplankton community identified in the eight sampled ponds comprises Rotifera species (11); Cladocera (7); Copepoda Cyclopoida (3); testate amoeba (3) and Anostraca (2) (Table 2). Anostraca was frequent in seven out of the eight sampled ponds; only in pond U7 there were no such large Branchiopoda. Among the cladocerans, the most frequent species was Moina minuta, followed by Simocephalus latirostris. Copepoda Cyclopoida and Rotifera species were less frequent (Table 2). Considering each pond, richness was very low ( 3 species in LG; 5 species in U6; 6 species in U1, U3, U4 and U5; 8 species in U2; and 13 species in U7). Concerning zooplankton biomass of all ponds, Anostraca was the most representative group, followed by Copepoda, Protozoa, Cladocera and Rotifera. Pond U6 had the highest total biomass, followed by LG and U4 (Table 2).

The first two axes of CCA applied to limnological variables and diversity of zooplankton community explained $91.5 \%$ of the data (Fig. 3). The most effective environmental variables in the axes were: T.S; T.P; T.N and conductivity. Anostraca was strongly related to $\mathrm{P}, \mathrm{N}$ and total solids, while Cladocera was slightly correlated with $\mathrm{N}$ and P. Copepoda and protozoa were very poorly related to conductivity, transparency and depth. The least effective environmental variables in the axes were depth and $\mathrm{pH}$, the latter being weakly related to the Rotifera.

The Pearson correlation coefficient between Anostraca and total solids, total phosphorus and total nitrogen was $0.1603 ; 0.7561$ and 0.7551 , respectively. Total solids are strongly related to

Table 1. Values of limnological variables of eight temporary ponds located in the State of Minas Gerais, Brazil. MD = mean depth; $\mathrm{Tp}=$ transparency (Secchi disk); Cond. = conductivity; $\mathrm{TN}=$ total nitrogen; $\mathrm{TP}=$ total phosphorus; $\mathrm{TS}=$ total suspended solids; IM = inorganic fraction of suspended solids; $\mathrm{OM}=$ organic fraction of suspended solids; $\mathrm{Chl}=$ chlorophyll a. Valores de variáveis limnológicas de oito lagoas temporárias localizadas no estado de Minas Gerais, Brasil. MD = profundidade média; Tp = transparência (disco de Secchi); Cond. = condutividade; $T N=$ nitrogênio total; $T P=$ fósforo total; $T S=$ sólidos suspensos totais; IM = fração inorgânica de sólidos em suspensão; $O M=$ fração orgânica de sólidos em suspensão; $C h l=$ clorofila a.

\begin{tabular}{ccccccccccc}
\hline & $\begin{array}{c}\text { MD } \\
\mathbf{c m}\end{array}$ & $\begin{array}{c}\text { TP } \\
\mathbf{c m}\end{array}$ & $\mathbf{p H}$ & $\begin{array}{c}\text { Condut. } \\
\boldsymbol{\mu S} / \mathbf{c m}\end{array}$ & $\begin{array}{c}\mathbf{T N} \\
\boldsymbol{\mu g} / \mathbf{L}\end{array}$ & $\begin{array}{c}\text { TP } \\
\boldsymbol{\mu g} / \mathbf{L}\end{array}$ & $\begin{array}{c}\mathbf{T S} \\
\mathbf{m g} / \mathbf{L}\end{array}$ & $\begin{array}{c}\mathbf{I M} \\
\mathbf{m g} / \mathbf{L}\end{array}$ & $\begin{array}{c}\mathbf{O M} \\
\mathbf{m g} / \mathbf{L}\end{array}$ & $\begin{array}{c}\mathbf{C h l} \\
\boldsymbol{\mu g} / \mathbf{L}\end{array}$ \\
\hline $\mathbf{U 1}$ & 25 & 5 & 5.9 & 18.9 & $2233 \pm 66.7$ & $133.3 \pm 5.0$ & $225.3 \pm 9.3$ & $198.7 \pm 2.8$ & $26.6 \pm 1.5$ & $0.025 \pm 0.004$ \\
$\mathbf{U} 2$ & 30 & 5 & 7.3 & 58.4 & $2300 \pm 33.3$ & $92.5 \pm 0.8$ & $136.5 \pm 6.3$ & $122.3 \pm 2.9$ & $14.2 \pm 1.9$ & $0.067 \pm 0.007$ \\
$\mathbf{U 3}$ & 35 & 5 & 6.6 & 42.7 & $4450 \pm 50.0$ & $110.0 \pm 1.7$ & $47.5 \pm 2.0$ & $39.5 \pm 1.4$ & $8.0 \pm 1.0$ & $0.018 \pm 0.004$ \\
$\mathbf{U} 4$ & 15 & 5 & 7.2 & 53.3 & $4733 \pm 33.3$ & $147.5 \pm 4.2$ & $59.9 \pm 1.8$ & $52.3 \pm 0.7$ & $7.6 \pm 0.3$ & $0.123 \pm 0.012$ \\
$\mathbf{U 5}$ & 20 & 7 & 6.6 & 39.1 & $2216 \pm 50.0$ & $88.3 \pm 3.3$ & $19.9 \pm 0.8$ & $15.4 \pm 2.2$ & $4.5 \pm 1.1$ & $0.010 \pm 0.004$ \\
$\mathbf{U 6}$ & 30 & 5 & 6.6 & 32.5 & $2083 \pm 50.0$ & $90.8 \pm 0.8$ & $37.7 \pm 1.9$ & $31.2 \pm 0.9$ & $6.5 \pm 0.5$ & $0.037 \pm 0.005$ \\
$\mathbf{U 7}$ & 10 & 10 & 6.7 & 33.8 & $3000 \pm 33.3$ & $110.8 \pm 0.8$ & $39.4 \pm 2.2$ & $25.0 \pm 1.0$ & $14.4 \pm 0.6$ & $0.052 \pm 0.007$ \\
$\mathbf{L G}$ & 8 & 8 & 6.6 & 30.8 & $*$ & $1714.2 \pm 4.2$ & $833.9 \pm 4.3$ & $707.5 \pm 2.6$ & $126.4 \pm 2.0$ & $0.019 \pm 0.005$ \\
\hline
\end{tabular}


Table 2. Numerical densities (ind. $\left./ \mathrm{m}^{3}\right)$, biomass - numbers in brackets $\left(\mu \mathrm{gDW} / \mathrm{m}^{3}\right)$, occurrence frequency $(\mathrm{OF} \%)$ and frequency (Freq.) classification of species of the zooplankton community from eight temporary ponds located in the State of Minas Gerais (Urucuia - U1 to U7, and Lagoa Grande - LG), Brazil. VF = very common; F = frequent; LF = less frequent; $\mathrm{S}=$ sporadic. Densidades numéricas (ind. $/ \mathrm{m}^{3}$ ), biomassa - números entre parênteses $\left(\mu \mathrm{gPS} / \mathrm{m}^{3}\right)$, frequências de ocorrência (OF \%) e classificação da frequência (Freq.) das espécies da comunidade zooplanctônica de oito lagoas temporárias localizadas no Estado de Minas Gerais (Urucuia - U1 a U7, e Lagoa Grande - LG), Brasil. VF= muito frequente; $F=$ frequente; $L F=$ pouco frequente; $S=$ esporádica.

\begin{tabular}{|c|c|c|c|c|c|c|c|c|c|c|}
\hline & U1 & U2 & U3 & U4 & U5 & U6 & U7 & LG & $\mathrm{OF} \%$ & Freq. \\
\hline \multicolumn{11}{|l|}{ Anos traca } \\
\hline Dendrocephalus brasiliensis & & $\begin{array}{c}145 \\
(27405)\end{array}$ & $\begin{array}{c}86 \\
(43000)\end{array}$ & $\begin{array}{c}108 \\
(183600)\end{array}$ & $\begin{array}{c}45 \\
(13500)\end{array}$ & $\begin{array}{c}117 \\
(257000)\end{array}$ & & & 75,0 & F \\
\hline Dendrocephalus thieryi & $\begin{array}{c}58 \\
(106140) \\
\end{array}$ & & & & & & & $\begin{array}{c}230 \\
(391000)\end{array}$ & 12,5 & S \\
\hline \multicolumn{11}{|l|}{ Cladocera } \\
\hline Alona glabra & & & & & & & $\begin{array}{c}250 \\
(328)\end{array}$ & & 12,5 & S \\
\hline Ceriodaphnia cornuta cornuta & & & & & & & $\begin{array}{l}700 \\
(906)\end{array}$ & & 12,5 & S \\
\hline Ceriodaphnia cornuta righaudi & & & & $\begin{array}{c}2 \\
(6)\end{array}$ & & & & & 12,5 & S \\
\hline Ilyocryptus spnifer & & & & & $\begin{array}{c}42 \\
(72)\end{array}$ & & $\begin{array}{r}500 \\
(818)\end{array}$ & & 25,0 & LF \\
\hline Macrothrix squamosa & & & & & & & $\begin{array}{c}300 \\
(460)\end{array}$ & & 12,5 & S \\
\hline Moina minuta & $\begin{array}{r}840 \\
(941)\end{array}$ & $\begin{array}{c}157 \\
(179)\end{array}$ & $\begin{array}{c}900 \\
(1117)\end{array}$ & $\begin{array}{c}48 \\
(58)\end{array}$ & $\begin{array}{l}1000 \\
(1118)\end{array}$ & & $\begin{array}{l}4719 \\
(5261)\end{array}$ & $\begin{array}{l}3200 \\
(3569)\end{array}$ & 87,5 & VF \\
\hline Simocephalus latirostris & & $\begin{array}{c}57 \\
(213) \\
\end{array}$ & $\begin{array}{l}20 \\
(75)\end{array}$ & & $\begin{array}{c}42 \\
(157)\end{array}$ & & $\begin{array}{c}990 \\
(3678) \\
\end{array}$ & & 50,0 & $\mathrm{~F}$ \\
\hline \multicolumn{11}{|l|}{$\begin{array}{l}\text { Copepoda } \\
\text { Cyclopoida }\end{array}$} \\
\hline Mesocyclops longisetus & & & & $\begin{array}{l}49,3 \\
(54)\end{array}$ & & & & & 12,5 & S \\
\hline Thermocyclops decipiens & & $\begin{array}{c}139 \\
(278)\end{array}$ & $\begin{array}{l}330 \\
(769)\end{array}$ & & & & $\begin{array}{c}820 \\
(1862)\end{array}$ & & 37,5 & LF \\
\hline Thermocyclops minutus & $\begin{array}{c}230 \\
(390)\end{array}$ & $\begin{array}{r}2899 \\
(3858)\end{array}$ & & & & & & & 25,0 & LF \\
\hline Young (nauplii/copepodite) & & & & & $\begin{array}{l}169000 \\
(73975) \\
\end{array}$ & $\begin{array}{r}48750 \\
(49824) \\
\end{array}$ & & $\begin{array}{r}160 \\
(187) \\
\end{array}$ & 37,5 & LF \\
\hline \multicolumn{11}{|l|}{ Rotifera } \\
\hline Asplanchna sieboldi & & & & $\begin{array}{c}5 \\
(8,9)\end{array}$ & & & $\begin{array}{l}100 \\
(194)\end{array}$ & & 25,0 & LF \\
\hline Brachionus angularis & & & & & & $\begin{array}{l}2250 \\
(450)\end{array}$ & & & 12,5 & S \\
\hline Brachionus calyciflorus & & $\begin{array}{c}157 \\
(31,4)\end{array}$ & & & & & & & 12,5 & S \\
\hline Lecane bulla & & & & & $\begin{array}{c}1250 \\
(329,5)\end{array}$ & & & & 12,5 & S \\
\hline Lecane curvicornis & $\begin{array}{c}340 \\
(70,4)\end{array}$ & & & & & & $\begin{array}{c}500 \\
(103,5)\end{array}$ & & 25,0 & LF \\
\hline Lecane furcata & & & & & & & $\begin{array}{c}230 \\
(36,7)\end{array}$ & & 12,5 & LF \\
\hline Lecane lunaris & $\begin{array}{c}800 \\
(166)\end{array}$ & & $\begin{array}{l}1200 \\
(249)\end{array}$ & & & & $\begin{array}{l}1900 \\
(393)\end{array}$ & & 37,5 & LF \\
\hline Lecane papuana & & $\begin{array}{l}1600 \\
(192)\end{array}$ & & & & & & & 12,5 & S \\
\hline Plathionus patulus & & $\begin{array}{l}900 \\
(435)\end{array}$ & $\begin{array}{l}1000 \\
(483)\end{array}$ & & & & $\begin{array}{c}500 \\
(242)\end{array}$ & & 37,5 & $\mathrm{LF}$ \\
\hline Polyarthra aff vulgaris & $\begin{array}{c}507 \\
(312)\end{array}$ & & & & & & $\begin{array}{c}702 \\
(432)\end{array}$ & & 25,0 & LF \\
\hline Bdelloidea & & & & $\begin{array}{c}2 \\
(1,8) \\
\end{array}$ & & $\begin{array}{r}58500 \\
(11285) \\
\end{array}$ & & $\begin{array}{r}4000 \\
(880) \\
\end{array}$ & 37,5 & LF \\
\hline \multicolumn{11}{|l|}{ Protozoa } \\
\hline Arcella mitrata & & & & & & $\begin{array}{c}750 \\
(61425)\end{array}$ & & & 12,5 & S \\
\hline Difflugia corona & & & & & $\begin{array}{l}3750 \\
(9375)\end{array}$ & & & & 12,5 & S \\
\hline Difflugia oblonga & & & & & & $\begin{array}{c}750 \\
(20873)\end{array}$ & & & 12,5 & S \\
\hline Total Density & 2775 & 6054 & 3536 & 214 & 175129 & 111117 & 12211 & 7590 & & \\
\hline Total Biomass & 108019 & 32591 & 45693 & 183728 & 98526 & 400857 & 14714 & 395636 & & \\
\hline
\end{tabular}

Limnetica, 38(1): 189-211 (2019) 


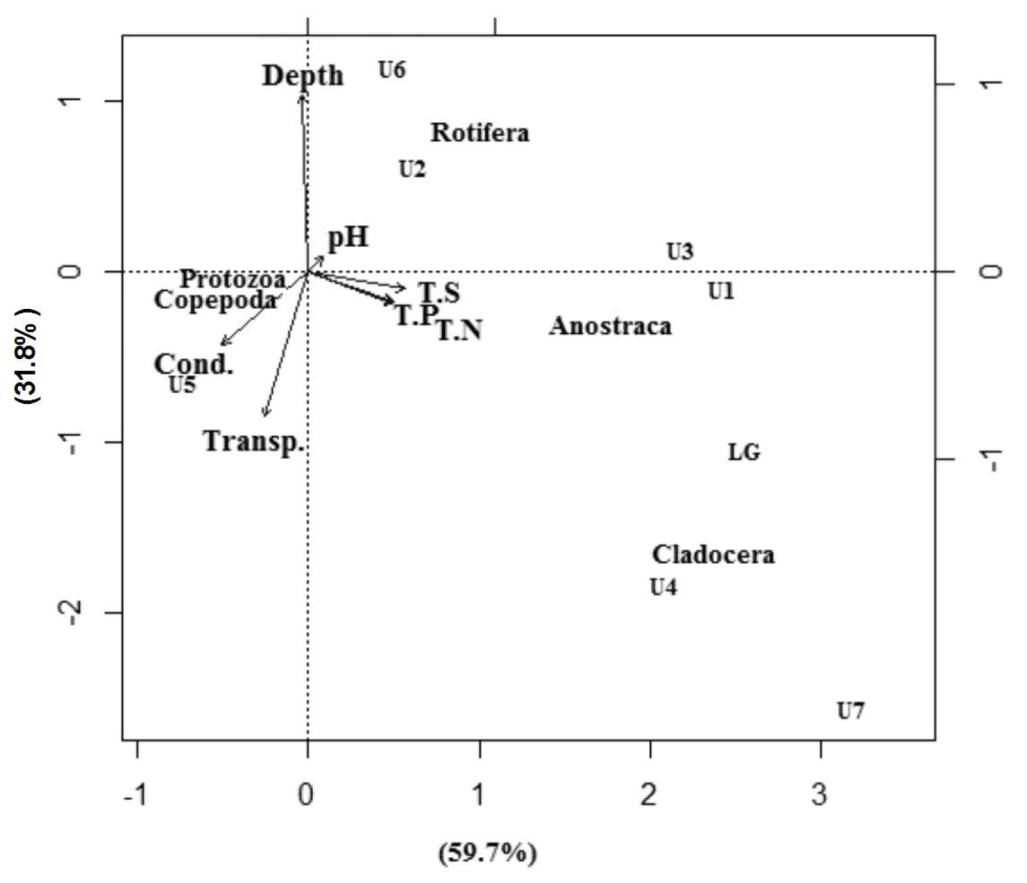

Figure 3. Canonical Correspondence Analysis (CCA) applied to limnological parameters and to the diversity of zooplankton groups from eight temporary ponds located in the State of Minas Gerais (Urucuia - U1 to U7, and Lagoa Grande - LG), Brazil. TS = total suspended solids; $\mathrm{TN}=$ total nitrogen; $\mathrm{TP}=$ total phosphorus; Transp. $=$ transparency; Cond $=$ conductivity. Análise de Correspondência Canônica (CCA) aplicada aos parâmetros limnológicos e à diversidade de grupos do zooplâncton de oito lagoas temporárias localizadas no Estado de Minas Gerais (Urucuia - Ul a U7, e Lagoa Grande - LG), Brasil. TS = sólidos suspensos totais; TN = nitrogênio total; $T P=$ fósforo total; Transp. = transparência; Cond = condutividade.

organic and inorganic matter, being this correlation of 0.9074 and 0.9988 . For Cladocera, the correlations were -0.2320 with total solids, 0.2352 with total phosphorus and 0.2300 with total nitrogen. For Copepoda and Protozoa, the correlation coefficient was close to zero. For Copepoda, the variables closest to presenting a tendency to correlation were, transparency $(0.0813)$ and depth $(0.0366)$, as well as for protozoa, transparency (0.0517) and depth (0.0685). Rotifera had a low correlation coefficient $(0.3200)$ with depth, and a relation with negative tendency for the other variables (Table S1, see supplementary information available at http://www.limnetica. net/en/limnetica)

Although cladocerans and rotifers may have sexual or asexual reproduction depending on environmental pressures, in the samples of this study, sexual females (ephippial) were absent for all species, therefore they had only asexual reproduction.
The dendrogram generated by multivariate cluster analysis with the distances between zooplankton species (Fig. 4), grouping them according eight functional traits (Table 3), resulting in the following five functional groups. G1: large pelagic herbivores, suspension feeders, ingesting food particles from 60 to $300 \mu \mathrm{m}$, the unique group with only one sexual reproduction per flood period, and mean duration of embryonic development (tED) of 1.4 days (D. brasiliensis and $D$. thieryi). G2: small littoral omnivores that capture small food particles $(0.2-10 \mu \mathrm{m})$ with pseudopods, with asexual reproduction by bipartition (many division cycles per flood period) and low tED $(<0.2$ days) (testated amoeba species). G3: small pelagic or littoral herbivores, mostly ciliate suspensivorous, ingesting small food particles $(10-15 \mu \mathrm{m})$, many asexual reproduction cycles per flood period and fast embryonic development, from 0.6 to 1.0 days (all rotifers except Asplanchna sieboldi). G4: medium pelagic or 
Table 3. Functional traits used for species of the zooplankton community of eight temporary ponds located in the State of Minas Gerais, Brazil. $\mathrm{BL}=$ body length $(\mathrm{mm}) ; \mathrm{TG}=$ trophic group $(\mathrm{H}=$ herbivore; $\mathrm{O}=$ omnivore; $\mathrm{C}=$ carnivore; $\mathrm{O} / \mathrm{C}=$ omnivore/ carnivore; $\mathrm{O} / \mathrm{H}=$ omnivore/ herbivore $) ; \mathrm{FH}=$ food habit $(\mathrm{SF}=$ suspension feeders; $\mathrm{Scr}=$ scraper; Rap = raptorial; Suc = sucking; Asp. = aspiration; $\mathrm{P}=$ pseudopodia $) ; \mathrm{R}=$ reproduction $(\mathrm{Ax}=$ asexual; $\mathrm{Sx}=$ sexual $) ; \mathrm{FSR}=$ food size range; Hab $=$ Habitat $(\mathrm{Lit}=$ littoral; $\mathrm{Pel}=$ pelagic); $\mathrm{NR} / \mathrm{F}=$ number of reproductions per flood period $(\mathrm{R} 1=$ only one reproduction; $\mathrm{R}+=$ several reproductions $)$; $\mathrm{tED}=$ duration of embryonic development in days. Traços funcionais utilizados para as espécies da comunidade zooplanctônica de oito lagoas temporárias localizadas no Estado de Minas Gerais, Brasil. $B L=$ comprimento do corpo (mm); $T G=$ grupo trófico $(H=$ herbívoro; $O=$ onivoro; $C=$ carnivoro; $O / C=$ onivoro/carnivoro; $O / H=$ onivoro/herbivoro); $F H=$ hábito alimentar $(S F=$ suspensivoro; $S c r$ $=$ raspador; Rap = raptorial; Suc = sugador; Asp. =aspirador; $P=$ pseudópodes $) ; R=$ reprodução $(A x=$ assexuada; $S x=\operatorname{sexuada)}$; $F S R=$ intervalo de tamanho do alimento; Hab = Habitat $($ Lit = litoral; $\mathrm{Pel}=$ pelágico); NR/F = número de reproduções por periodo de cheia $(R I=$ apenas uma reprodução; $R+=$ várias reproduções; $t E D=$ duração do desenvolvimento embrionário em dias.

FUNCTIONAL TRAITS

\begin{tabular}{|c|c|c|c|c|c|c|c|c|}
\hline & BL & TG & FH & $\mathbf{R}$ & FSR & Hab & NR/F & tED \\
\hline \multicolumn{9}{|l|}{ Anostraca } \\
\hline Dendrocephalus brasiliensis & 17.02 & $\mathrm{H}$ & $\mathrm{SF}$ & $\mathrm{Sx}$ & $60-300 \mu \mathrm{m}$ & Pel & R1 & 1.4 \\
\hline Dendrocephalus thieryi & 21.00 & $\mathrm{H}$ & SF & Sx & $60-300 \mu \mathrm{m}$ & Pel & R1 & 1.4 \\
\hline \multicolumn{9}{|l|}{ Cladocera } \\
\hline Alona glabra & 0.29 & $\mathrm{H}$ & Scr & $\mathrm{Ax}$ & $10-15 \mu \mathrm{m}$ & Lit & $\mathrm{R}^{+}$ & 1.6 \\
\hline C. cornuta cornuta & 0.49 & $\mathrm{H}$ & $\mathrm{SF}$ & $\mathrm{Ax}$ & $15-40 \mu \mathrm{m}$ & Pel & $\mathrm{R}^{+}$ & 1.7 \\
\hline C. cornuta righaudi & 0.54 & $\mathrm{H}$ & $\mathrm{SF}$ & $\mathrm{Ax}$ & $15-40 \mu \mathrm{m}$ & Pel & $\mathrm{R}+$ & 1.7 \\
\hline Ilyocryptus spnifer & 0.62 & $\mathrm{H}$ & Scr & $\mathrm{Ax}$ & $15-40 \mu \mathrm{m}$ & Lit & $\mathrm{R}+$ & 2.5 \\
\hline Macrothrix squamosa & 0.58 & $\mathrm{H}$ & Scr & $\mathrm{Ax}$ & $15-40 \mu \mathrm{m}$ & Lit & $\mathrm{R}+$ & 1.7 \\
\hline Moina minuta & 0.42 & $\mathrm{H}$ & $\mathrm{SF}$ & $\mathrm{Ax}$ & $15-40 \mu \mathrm{m}$ & Pel & $\mathrm{R}+$ & 1.4 \\
\hline Simocephalus latirostris & 1.40 & $\mathrm{H}$ & $\mathrm{SF}$ & $\mathrm{Ax}$ & $40-100 \mu \mathrm{m}$ & Pel & $\mathrm{R}^{+}$ & 1.8 \\
\hline \multicolumn{9}{|l|}{ Copepoda Cyclopoida } \\
\hline Mesocyclops longisetus & 1.08 & $\mathrm{OC}$ & Rap & Sx & $40-100 \mu \mathrm{m}$ & Lit & $\mathrm{R}+$ & 1.8 \\
\hline Thermocyclops decipiens & 0.97 & $\mathrm{OH}$ & Rap & Sx & $15-40 \mu \mathrm{m}$ & Lit & $\mathrm{R}^{+}$ & 1.9 \\
\hline Thermocyclops minutus & 0.72 & $\mathrm{OH}$ & Rap & Sx & $15-40 \mu \mathrm{m}$ & Lit & $\mathrm{R}+$ & 1.9 \\
\hline \multicolumn{9}{|l|}{$\overline{\text { Rotifera }}$} \\
\hline Asplanchna sieboldi & 0.68 & $\mathrm{C}$ & Asp & $\mathrm{Ax}$ & $40-100 \mu \mathrm{m}$ & Pel & $\mathrm{R}+$ & 1.0 \\
\hline Brachionus angularis & 0.12 & $\mathrm{H}$ & $\mathrm{SC}$ & $\mathrm{Ax}$ & $10-15 \mu \mathrm{m}$ & Pel & $\mathrm{R}^{+}$ & 0.9 \\
\hline Brachionus calyciflorus & 0.32 & $\mathrm{H}$ & $\mathrm{SC}$ & $\mathrm{Ax}$ & $10-15 \mu \mathrm{m}$ & Pel & $\mathrm{R}+$ & 0.9 \\
\hline Lecane bulla & 0.13 & $\mathrm{H}$ & $\mathrm{SC}$ & $\mathrm{Ax}$ & $10-15 \mu \mathrm{m}$ & Lit & $\mathrm{R}+$ & 0.6 \\
\hline Lecane curvicornis & 0.12 & $\mathrm{H}$ & $\mathrm{SC}$ & $\mathrm{Ax}$ & $10-15 \mu \mathrm{m}$ & Lit & $\mathrm{R}^{+}$ & 0.6 \\
\hline Lecane furcata & 0.11 & $\mathrm{H}$ & $\mathrm{SC}$ & $\mathrm{Ax}$ & $10-15 \mu \mathrm{m}$ & Lit & $\mathrm{R}^{+}$ & 0.6 \\
\hline Lecane lunaris & 0.12 & $\mathrm{H}$ & $\mathrm{SC}$ & $\mathrm{Ax}$ & $10-15 \mu \mathrm{m}$ & Lit & $\mathrm{R}^{+}$ & 0.6 \\
\hline Lecane papuana & 0.10 & $\mathrm{H}$ & $\mathrm{SC}$ & $\mathrm{Ax}$ & $10-15 \mu \mathrm{m}$ & Lit & $\mathrm{R}^{+}$ & 0.6 \\
\hline Plathionus patulus & 0.13 & $\mathrm{H}$ & $\mathrm{SC}$ & $\mathrm{Ax}$ & $10-15 \mu \mathrm{m}$ & Pel & $\mathrm{R}+$ & 1.0 \\
\hline Polyarthra aff vulgaris & 0.16 & $\mathrm{H}$ & Suc & $\mathrm{Ax}$ & $10-15 \mu \mathrm{m}$ & Pel & $\mathrm{R}^{+}$ & 0.9 \\
\hline Bdelloidea & 0.16 & $\mathrm{H}$ & $\mathrm{SC}$ & $\mathrm{Ax}$ & $10-15 \mu \mathrm{m}$ & Lit & $\mathrm{R}^{+}$ & 1.0 \\
\hline \multicolumn{9}{|l|}{$\overline{\text { Protozoa }}$} \\
\hline Arcella mitrata & 0.16 & $\mathrm{O}$ & $\mathrm{P}$ & $\mathrm{Ax}$ & $0.2-10 \mu \mathrm{m}$ & Lit & $\mathrm{R}+$ & $<0.2$ \\
\hline Difflugia corona & 0.19 & $\mathrm{O}$ & $\mathrm{P}$ & $\mathrm{Ax}$ & $0.2-10 \mu \mathrm{m}$ & Lit & $\mathrm{R}+$ & $<0.2$ \\
\hline Difflugia oblonga & 0.21 & $\mathrm{O}$ & $P$ & $\mathrm{Ax}$ & $0.2-10 \mu \mathrm{m}$ & Lit & $\mathrm{R}^{+}$ & $<0.2$ \\
\hline
\end{tabular}

Limnetica, 38(1): 189-211 (2019) 


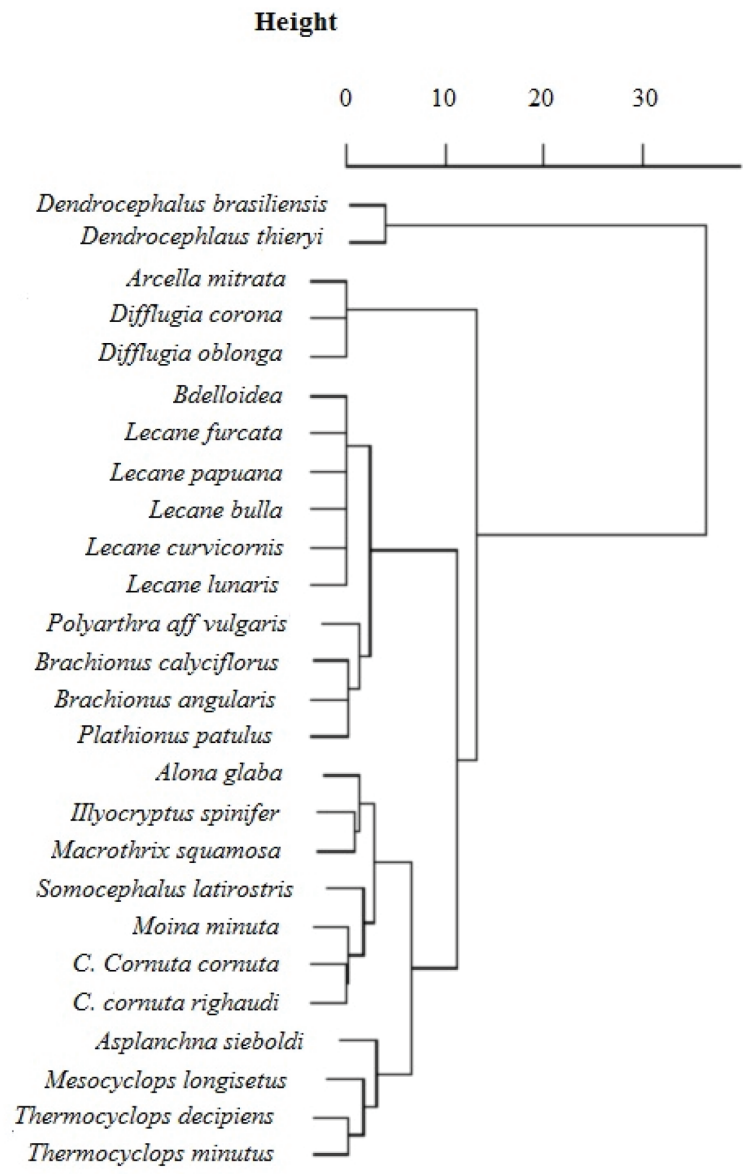

Figure 4. Dendrogram generated by multivariate Cluster analysis with the distances between zooplankton species, and grouping them according eight functional traits. Dendrograma gerado por análise multivariada de Cluster com as distâncias entre espécies zooplanctônicas, agrupando-as de acordo com oito traços funcionais.

littoral herbivores, suspensivorous or scrapers, ingesting food particles from 10 to $100 \mu \mathrm{m}$, with many asexual reproductive cycles per flood period, and tED from 1.4 to 2.5 days (all Cladocera). G5: pelagic or littoral raptorial predators (predominantly omnivorous or carnivorous), ingesting food particles from 15 to $100 \mu \mathrm{m}$, with many asexual or sexual reproduction cycles per flood period, and tED from 1.0 to 1.9 days (all Copepoda and the rotifer Asplanchna sieboldi).

From data of the relative abundance of the five functional groups in each temporary pond (Table 4), a CCA relating this abundance to the limnolog- ical parameters (Fig. 5) shows that $80.8 \%$ of the data was explained by the first two axes, where G3 was correlated to ponds' mean depth; G4 was related to water transparency, total suspended solids, total $\mathrm{P}$ and $\mathrm{N}$; G5 was correlated to conductivity and $\mathrm{pH}$; and $\mathrm{G} 1$ and $\mathrm{G} 2$ did not present direct correlation with any analyzed variables.

Values of two indexes, Shannon Diversity $\left(\mathrm{H}^{\prime}\right)$ and Functional Diversity (FDis), calculated for the zooplankton community of the studied ponds are, respectively: $1.04 / 0.18$ for $\mathrm{U} 1$; $1.03 / 0.31$ for $\mathrm{U} 2 ; 0.95 / 0.19$ for $\mathrm{U} 3 ; 1.43 / 0.38$ for $\mathrm{U} 4 ; 1.28 / 0.28$ for $\mathrm{U} 5 ; 1.30 / 0.33$ for $\mathrm{U} 6 ; 1.36 / 0.30$ for U7; and 1.03/0.15 for LG. It can be observed $\mathrm{H}$ ' ranging from 0.95 to 1.43 and low values of FDis $(0.15$ to 0.38$)$. Ponds also presented low values of species richness (3 to 13 species).

\section{DISCUSSION}

\section{Characteristics of the studied ecosystems}

The results of this study indicate that because all the sampled ephemeral ecosystems are small, very shallow (mean depth from 8 to $30 \mathrm{~cm}$ ) and are rain-fed ponds isolated from another source of water, there is a high suspension of sediment particles (which causes low transparency - 2 a $10 \mathrm{~cm}$ ), especially the inorganic ones (from $63.5 \%$ in U7 to $89.6 \%$ in U2). Most of the studied ponds have little or no vegetation in their surroundings, which may be related to high amounts of suspended solids and inorganic matter, which was also observed by Sahuquillo et al. (2012). Inorganic particles adsorb phosphorous reducing its bioavailavility in water. Due to this, although we found high amounts of total phosphorus (and also total nitrogen), comparable to eutrophic lakes, planktonic chlorophyll $a$ was very low and not correlated with total nutrients, probably due to low light penetration in these environments, which limits the development of the phytoplanktonic community. The organic fraction of suspended material was low in all ponds, but proportionally higher in those covered by grasses (U7; LG) or with more vegetation in its surroundings (U5). This probably also applies to the sediment due to the short hydroperiod of ponds (about three months per year) since desiccation 


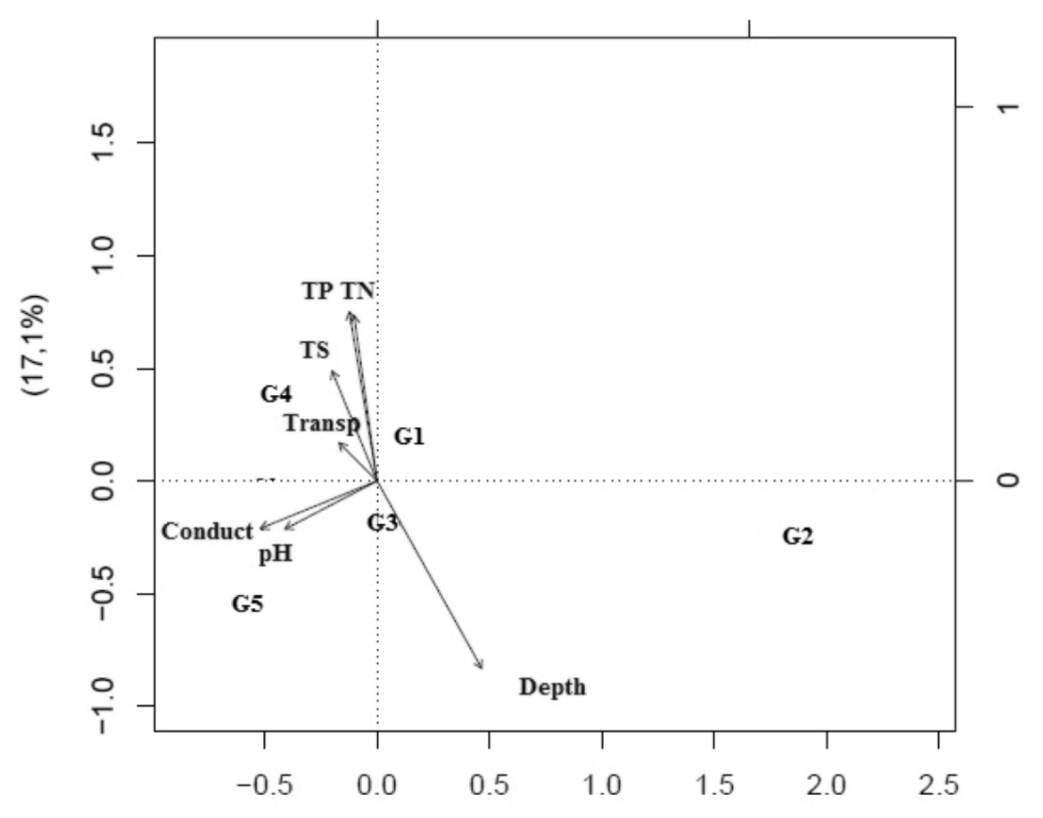

$(63,7 \%)$

Figure 5. Canonical Correspondence Analysis (CCA) applied to limnological parameters and relative abundances of five functional groups (G1 to G5) of the zooplankton community from eight temporary ponds located in the State of Minas Gerais, Brazil. TS = total suspended solids; $\mathrm{TN}=$ total nitrogen; $\mathrm{TP}=$ total phosphorus; Transp. $=$ transparency; Conduct $=$ conductivity. Análise de Correspondência Canônica (CCA) aplicada aos parâmetros limnológicos e às abundâncias relativas de cinco grupos funcionais (G1 a G5) da comunidade zooplanctônica de oito lagoas temporárias localizadas no Estado de Minas Gerais, Brasil. TS = sólidos suspensos totais; $T N=$ nitrogênio total; $T P=$ fósforo total; Ttransp. $=$ transparência $;$ Conduc $=$ condutividade.

has important effects on biogeochemical transformations. A threshold of $5 \%$ of organic matter in the sediment separates the temporary lagoons with a short hydroperiod of those with a long hydroperiod. The shallower the pond, the shorter the hydroperiod and the lower the organic matter that the sediment contains, the lesser the total nitrogen proportion with respect to total phosphorus (Sahuquillo et al., 2012). These authors also state that an important loss of $\mathrm{N}$ occurs during desiccation through mineralization, ammonia volatilization and the sequential nitrification/denitrification pathway and that the increase of organic phosphorus mineralization in dry sediments does not represent a loss of $\mathrm{P}$ from the system, since it remains adsorbed to the soil, which also leads to a low TN/TP ratio. Despite this, all ponds studied here had high amounts of total nitrogen and a high proportion with respect to total phosphorus, at least in the water column. As these ponds are widely used in the region for cattle watering, which in addition to trampling suspending the sediment possibly contributes to increasing the nitrogen concentration in the water with their feces and urine. In addition, the high temperatures of these shallow environments may favor the processes of releasing nutrients from sediment.

The $\mathrm{pH}$ did not vary much between the studied ponds, ranging from slightly acid to neutral (5.9 to 7.3), close to the most continental water bodies whose $\mathrm{pH}$ ranges from 6.0 to 8.5 (Margalef, 1983; Kalff, 2002). The decomposition of the organic matter decreases the water $\mathrm{pH}$. However, as the amount of organic matter in these environments is low, probably its $\mathrm{pH}$ is more related to geological characteristics and soil type sites. The ponds are located in a Brazilian savanna ("cerrado") region, near the semi-arid region of northeast Brazil, where soils tend to be sandy with a higher acidity. Similarly, conductivity does not seem to be associated with the decomposition in these environments, since 
Table 4. Relative abundance (\%) of the functional groups (G1 to G5) defined for the zooplankton community of eight temporary ponds located in the State of Minas Gerais, Brazil. Ponds U1 to U7 are located in the city of Urucuia and LG, in the city of Lagoa Grande. Abundância relativa (\%) dos grupos funcionais (G1 a G5) definidos para a comunidade zooplanctônica de oito lagoas temporárias localizadas no Estado de Minas Gerais, Brasil. As lagoas U1 a U7 são localizadas na cidade de Urucuia e LG, na cidade de Lagoa Grande.

\begin{tabular}{ccccccccc}
\hline & U1 & U2 & U3 & U4 & U5 & U6 & U7 & LG \\
\hline G1 & 16.67 & 12.50 & 16.67 & 16.67 & 16.67 & 20.00 & 0.00 & 33.33 \\
G2 & 0.00 & 0.00 & 0.00 & 0.00 & 16.67 & 40.00 & 0.00 & 0.00 \\
G3 & 50.00 & 37.50 & 33.33 & 16.67 & 16.67 & 40.00 & 38.45 & 33.33 \\
G4 & 16.67 & 25.00 & 33.33 & 33.33 & 50.00 & 0.00 & 46.15 & 33.33 \\
G5 & 16.67 & 25.00 & 16.67 & 33.33 & 0.00 & 0.00 & 15.40 & 0.00 \\
\hline
\end{tabular}

values did not differ much between the ponds and were not very high, even in ponds covered by vegetation such as U7. Thus, conductivity should be more associated with the drainage basin phenomena, such as the nature of rocks and soil of the basin, the type of vegetation and the effects of rainwater leaching. Ions concentration in water is also strongly dependent on the temperature, which is quite high in these ponds, often exceeding $30^{\circ} \mathrm{C}$. In fact, in tropical regions, conductivity of aquatic environments is more related to the geochemical characteristics of the region where they are located and to the climatic conditions (dry and rainy season) than with the trophic state, as in temperate regions, which have in general higher conductivity values (Esteves, 1988).

Therefore, temporary aquatic ecosystems have unique characteristics connected to dry periods and rainy seasons that influence the fluctuation of the water level and, as a consequence, have their physical and chemical characteristics altered (Maltchik et al., 1999). Important factors such as rainfall and evaporation rates have a significant impact on the dynamics of these ecosystems (Tundisi \& Matsumura Tundisi, 2008).

\section{Zooplankton Community}

\section{Taxonomic diversity and biomass}

In the present study, we found a very low diversity of the zooplankton community in all ponds studied. Species richness in each pond ranged from 4 to 13, and we found only 27 taxa considering all ponds, recorded among Anostraca, Cladocera, Copepoda, Rotifera and Protozoa. Other studies in Brazilian aquatic ecosystems recorded much higher zooplankton species richness both in temporary and in perennial environments (reservoirs and rivers). Passos (2012) found 20 to 23 taxa in three shallow temporary ponds in the municipality of Urucuia, Minas Gerais (near to the ponds of this study); Passos (2017) registered 68 taxa for a total of 25 shallow temporary ponds from Minas Gerais (municipalities of Urucuia, Lagoa Grande and Pirapora) studied during three consecutives years (2015 to 2017); Moreira et al. (2016) registered 29 taxa in a shallow high-elevation temporary pond in Minas Gerais; in São Paulo State, Melão (1997) found 28 taxa in a shallow permanent oligotrophic reservoir and Santos (2010) found richness ranging from 17 to 36 in three large big reservoirs of Tietê River (São Paulo); Silva (2015), in a review of studies done in large amazonic rivers, reported 143 species in the Madeira River and 129 species in the Xingu River. It is possible, however, that our results underestimate the real richness of zooplankton species in the studied ponds, since we did a single sampling campaign and resistance eggs of the species from such temporary ecosystems can hatch in different times (Passos, 2017). Even so, it seems possible to identify a pattern in the coexistence between zooplanktonic species. It seems clear that larger filtering animals, such as large cladocerans species, are absent in the 
presence of Dendrocephalus species or may coexist with them but with low population densities. Passos (2017), studying 25 temporary ponds of Minas Gerais, recorded the occurrence of Copepoda Calanoida in ponds with absence or small population density of Dendrocephalus brasilienses. On the other hand, smaller cladocerans seem to coexist well with these large Branchiopoda. The most successful Cladocera species in the sampled environments in the present study is Moina minuta, both in terms of frequency of occurrence and number of individuals. Passos (2017) also recorded the occurrence of this species in $100 \%$ of the 25 environments of his study, in addition to presenting the highest numerical densities. Among the Copepoda, the Cyclopoida are predominant in the temporary ponds studied, particularly the species of Thermocyclops. Passos (2017) also recorded Thermocyclops decipiens and $T$. minutus as the most frequent. Rotifera species are more numerous, but L. lunaris and Plathionus patulus are some of the most frequent in these temporary environments, which was also found by Passos (2017).

Temporary ecosystems are generally small, shallow and isolated (Pérez-Bilbao et al., 2015), which results in a relatively low biodiversity (Zacharias et al., 2007; Crispim \& Freitas, 2005). In a study of 29 temporary ponds in the UK, divided into permanent (which have well defined periods of flood and dry) and temporary semi-permanent (which exceptionally dry up and can spend years in flood periods), the authors found a greatly reduced number of species in the semi-permanent temporary ponds compared to the others (Collinson et al., 1995). These authors state that temporary environments are ecosystems of rare species, with much endemism, and report that, in addition to harboring rare plants and amphibians, $25 \%$ of endangered freshwater invertebrate species inhabit temporary waters. Thus, temporary aquatic ecosystems have a great evolutionary and ecological importance, housing communities which are adapted physiologically and that develop efficient and diversified mechanisms for their colonization, permanence and reproduction.

In the present work, we found two Anostraca species, both from the Thamnocephalidae family: Dendrocephalus brasiliensis (ponds U2 to U6) and Dendrocephalus thieryi (ponds U1 and LG). In Brazil, so far, D. thieryi has had an occurrence recorded only for the municipality of Buritizeiro, MG (Rabet, 2006); therefore a new record was made of this species in the present study. The populations of the sampled Dendrocephalus had homogeneous ages (male and female adults) as diapause eggs hatch all at the same time when the pond is filled during the rainy season (Lopes, 2007).

Rotifera had the highest species richness in the studied ponds, as occurs in most aquatic ecosystems, especially in tropical regions (Rocha et al., 1995), which is justified by the high population growth rates of r-strategist species, and also adaptation to the periodic alterations of these ecosystems (Allan, 1976). The other groups had lower species richness and their frequency of occurrence was generally low. Moreover, most of these species were classified as sporadic and infrequent. Moina minuta, D. brasiliensis, and Simocephalus latirostris had a higher frequency and M. minuta was the most frequent species in all the ponds sampled. This is explained by the success in the colonization strategies of these species, mainly the production of dormant eggs that resist the desiccation periods (Passos, 2017).

Related to Protozoa, probably the number of species is underestimated as the collection and fixation methodologies used in this study were not targeted at such organisms, and certainly promoted the disruption of these unicellular individuals. Only the carapaces of testate amoeba remained, which were recorded in this study.

Comparing the numerical densities of zooplankton in the eight studied ponds, we observed that in U7, where Dendrocephalus species are absent, there is a significant increase of all species of Cladocera, especially M. minuta, Simocephalus latirostris and Ceriodaphnia cornuta, as well as adults of Copepoda Cyclopoida Thermocyclops decipiens. There is, therefore, clear evidence that Anostraca interfered in the species composition and in the population densities of the Cladocera, possibly due to the niche overlap. In addition, there are few large species of Cladocera, with a predominance of small species, able to feed on bacteria and debris, since phytoplankton is relatively scarce in these environ- 
ments. A larger Cladocera, Simocephalus latirostris, occurs in higher densities only in U7, probably due to the presence of aquatic plants, which occurs only in this pond.

Generally, the initial phase of an environment is dominated by larger species, with strategies of rapid colonization; and the other species, k-strategists, tend to colonize it later (Vanschoenwinkel et al., 2011). The Anostraca species are large and r-strategists and can be good competitors for resources as they are generalists, adapted to temporary ecosystems and fast hatching and, because of this, interfere in the population composition of the other community groups (Sarma \& Nandini, 2002). The zooplankton species of the temporary environments, however, have several strategies to avoid the niche overlap. One of them is the time lag in the hatching of resistance eggs of different zooplankton species (Passos, 2017), indicating a partition of niches between them, which would minimize the competition for resources, leading to the successful coexistence of these species in these temporary ecosystems.

The competition in these temporary ponds is intense, since the environmental conditions do not favor food abundance, especially phytoplankton due to low penetration of light. In the CCA, it can be observed that Anostraca and Cladocera (in lesser degree) are favored by parameters such as suspended solids, total nitrogen and phosphorus, which are indirectly related to the availability of food in the water column, such as debris and bacteria; Copepoda were slightly favored by variables (conductivity and transparency) that indicate better conditions for phytoplankton growth, from which their young phases feed and also the adults' prey.

In terms of biomass, Dendrocephalus species far exceeded the other groups in all ponds where they occur. In the absence of the Anostraca (pond U7), the cladocerans $M$. minuta and S. latirostris stand out for the high biomass, followed by the copepod T. decipiens. The increase of biomass of these species should be related to the greater availability of food particles, since there is no presence of the big anostracean competitors.

Since there are variations in the size of the species, and each one plays a different role in the ecosystem, biomass more effectively describes the zooplankton community (Melão \& Rocha, 2004), providing a perception of the trophic chain, energy and the actual contribution of each species to ecological processes (Begon \& Townsend, 2006). However, the real role of different species in ecosystems also depends on how efficient they are in renewing their biomass. Rotifers, for example, are small but have a high rate of renewal of their populations, that is, they have a high production/biomass ratio ( $\mathrm{P} / \mathrm{B}$ ratio). Other groups, such as Copepoda, for example, are larger and they have relatively high fecundity, but have a longer time of embryonic and post-embryonic development, which could result in lower population turnover rates compared to Cladocera or Rotifera. In the temporary ecosystems studied here, species that have higher population turnover rates have competitive advantages over others, since the hydroperiod is very short. These questions are very important in the ecology of zooplankton and completely unknown to Brazilian temporary ponds, and therefore more in-depth studies are needed.

\section{Functional Diversity}

Analyzing the functional diversity of the studied ponds indicated that the most determinant attributes to group the species were the trophic group and feeding habits. Although the attributes associated with reproduction were also important, food relationships were more decisive. In small environments, as specific as those sampled in this study, a certain convergence in the way of feeding is natural, especially due to scarcity or less variety of resources. In the sampled ponds, a predominance of species occurs with passive food capture, even if the search is active. Each type of feed has costs and benefits, and passive feeding allows for less energy expenditure, and a low risk of predator exposure. The size of food particles is also a criterion that can determine the grouping of species, and is directly related to food habits (Barnett et al., 2007). Herbivores, which were found in half of the functional groups of this study, tend to feed on smaller particles, which generally fluctuate in the aquatic environment, favoring passive feeding, while carnivores tend to feed on larger particles as they spend more energy 
on active feeding activities. The size of the food particles, therefore, can determine patterns of food strategies (Litchman, 2013).

In CCA, the functional groups correlated with environmental variables favor greater food availability. The group of raptorial predators (G5), are favored by the presence of prey, which in turn are more correlated to the variables that favor the growth of bacteria or phytoplankton like transparency and nutrients; the filter-feeders (especially G4) are favored by variables like TP, TN, TS that favor the presence of food in the water column (especially bacteria and mixotrophic nanoflagellates), and by an increase in light penetration, which increases phytoplankton growth.

Type of reproduction is important in temporary aquatic ecosystems, where species need to invest in strategies to ensure their permanence considering so many environmental oscillations. Reproduction occurs asexually (by parthenogenesis) in the flood period, but the stress caused when the environment is drying induces the formation of resistance eggs through sexual reproduction. In this functional trait, we considered the reproduction predominant for each species according to our samples, although there may be changes depending on the environmental conditions. Reproduction and growth rates of zooplankton community are a reflection of the ways of allocating energy (Litchman, 2013). Investing in growth or reproduction, for example, can be a decisive choice for species. In the zooplankton community this trade-off in investment is very clear: investment in reproduction implies a reduced growth and reduced lifetime. On the contrary, investment in growth automatically implies less longevity. Rotifers, for example, allocate more energy to reproduction, and fail to invest in body size and longevity. In contrast, copepods have defined stages of development, with stages of maturation and greater longevity, which implies in a lower risk of predation (Huntley \& Lopez, 1992). Moreover, concerning reproduction, a large number of offspring is directly related to a smaller body size of each individual of offspring, which results in the reduction in individual survival. This is a constant strategy for the permanence of species of the zooplankton community in the ecosystem (Litchman, 2013).
In the present study, the functional grouping of zooplankton community was very close to the taxonomic grouping, with the exception of G5 that grouped the three species of Copepoda Cyclopoida and one of Rotifera, mainly due to their similar eating habits (all are raptorial predators). This result may have been influenced by our choice of functional traits. However, it should be considered that the taxonomic classification of species is already based precisely on their morphological characteristics that are in some way related to the function performed by the species within the ecosystem (Silva, 2015). In general, natural selection tends to accentuate individual fitness, increasing the outcome of eating, survival and growth patterns within communities (Litchman, 2013). However, even if functional bias is similar to taxonomic grouping in many cases, it is important to know the functional attributes and also how they relate to the influences of the environment (Brasil \& Huszar, 2011).

The functional diversity analyzed in this study for the zooplankton community was generally low (from 0.15 to 0.38 in an index that ranges from 0 to 1.0) for all environments sampled. This may indicate a dominance of few functional attributes, which may be the result of the strong action of environmental filters in the community structure and would determine the occurrence of species with more similar functional characters. Low functional diversity of zooplankton can therefore inform us that important processes that occur in the community structure can be influenced by the action of physical and/or chemical characteristics or environmental filters that act on the species, promoting a confluence of the functional characteristics. But on the other hand it is also possible that this occurs due a great variation within each zooplankton species in order to adapt to environmental conditions.

In a large spatial scale study with mammals of tropical ecosystems, Safi et al. (2011) identified low functional diversity in relation to the taxonomic one and affirm that this is a common pattern in tropical regions, suggesting that there is a high number of ecologically similar species due to a smaller evolutionary history between them, which also shows a low phylogenetic diversity. Considering the few studies on zooplankton in 
tropical regions, it is too early to say that this also applies to this community.

In fact, maybe zooplanktonic community is not as good as phytoplankton in studies of functional diversity. For phytoplankton, this kind of study is already well established (Brasil \& Huszar, 2011), but it is not to zooplankton. As zooplankton presents several adaptation characters in individuals within the same species, it is difficult to choose traits that really differentiate them. For example, some cladocerans are larger in presence of invertebrate predators and smaller in the presence of visual predators (Caramujo et al., 1997; Crispim \& Boavida, 2001), so their length can change depending on the biological interactions; reproduction type (sexual or asexual) can also changes according environment conditions; fecundity also have great intraspecific differences, related, for example, to female size and food conditions (Vieira et al., 2011); food source can also present great variability. Anyway, it is not easy choose the right functional traits, and the wrong choice can lead to misinterpretations of the results.

Thus, although studies related to functional diversity have advanced in recent years, as far as zooplankton communities are concerned, much remains to be done. A barrier that may account for such a deficiency would be the lack of basic studies on the biology of organisms, since with more detailed and accurate information; a greater number of functional traits could be used. There are many important traits for each species, but measuring them all is not possible for many reasons. Many of the traits to be studied have already been suggested by Litchman et al. (2013). Thus, better and broader measures of functional diversity would be possible, which would further explain the ecological functions of organisms and their relationship with ecosystems.

Using various ways to measure diversity can help assess evolutionary and ecological processes that act on diversity. The biological diversity of ecosystems has been investigated in recent decades with the help of many ecological theories of communities that address spatial and temporal patterns, such as the ecological succession theory (Gleason, 1927) and the theory of island biogeography (Macarthur \& Wilson, 1967). In the theory of island biogeography, the research carried out by the authors is based on three observations: (1) Island communities are poorer in species than equivalent continental communities; (2) richness increases with island size; (3) richness decreases with increasing isolation of the island. Perhaps we can make an analogy here of the object of our study with islands as the temporary ponds studied in this research seem to follow the same premises of this theory: they are small and isolated ecosystems, which have a low diversity of species when compared to other ecosystems of continental waters.

Thus, the present study contributed to increasing knowledge on the taxonomic and functional diversity of the zooplankton community of temporary aquatic ecosystems in the Neotropics. Environmental conditions and very peculiar characteristics of these ephemeral ecosystems have resulted in low diversity indexes of the zooplankton community, where few species with similar ecological functions and similar strategies ensure establishment, permanence and reproduction in these environments, such as high reproduction capacity, rapid growth, short life cycles and type of feed. In addition, there was evidence that Anostraca species of the genus Dendrocephalus have an impact on the taxonomic composition and numerical density of the community, especially Cladocera.

\section{ACKNOWLEDGEMENTS}

The authors are thankful to the Coordination for the Improvement of Higher Level Education Personnel - CAPES and the National Council for Scientific and Technological Development CNPq, Brazil [grant number 305229/2016-8]. This study was supported by the São Paulo Research Foundation - FAPESP, Brazil [proc. numbers 2014/14139-3 and 2016/00753-7].

\section{REFERENCES}

ALLAN, J. D. 1976. Life history patterns in zooplankton. The American Naturalist, 110: 165-180. DOI: $10.1086 / 283056$

ATASHBAR, B., N. AGH, G. V. STAPPEN \& L. BELADJAL. 2014. Diversity and distribution 
patterns of large branchiopods (Crustacea: Branchiopoda) in temporary pools (Iran). Journal of Arid Environments, 111(2014): 27-34. DOI: 10.1016/j.jaridenv.2014.07.005

BARNETT, A. J., K. FINLAY \& B. E. BEISNER. 2007. Functional diversity of crustacean zooplankton communities: towards a trait-based classification. Freshwater Biology, 52(5): 796-813. DOI: 10.1111/j.1365-2427.2007. 01733.x

BARNETT, A. J., K. FINLAY \& B. E. BEISNER. 2013. Corrigendum Functional diversity of crustacean zooplankton communities: towards a trait-based classification. Freshwater Biology, 58: 1755-1765 DOI: 10.1111/ fwb. 12177

BEGON, M. \& C. R. TOWNSEND. 2006. Ecology: from individuals to ecosystems. Blackwell Publishing. Oxford. UK.

BERN, L. 1994. Particle selection over a broad size range by crustacean zooplankton. Freshwater Biology, 32(1): 105-112. DOI: 10.1111/j.1365-2427.1994.tb00870.x

BICUDO, C. E. D. M \& D. D. C. BICUDO. 2004. Amostragem em limnologia. Rima, São Carlos. Brazil.

BODGAN, K. G. \& J. J. GILBERT. 1987. Quantitative comparison of food niches in some freshwater zooplankton. Oecologia, 72(3): 331-340. DOI: 10.1007/BF00377560

BOTTRELL, H. H., A. DUNCAN, Z. M. GLIWICZ, E. GRYGIEREK, A. HERZING, A. HILLBRICHTLLKOWSKA, H. KURASAWA, P. LARSSON \& T. A. W'EGLENSKA. 1976. Review of some problems in zooplankton production studies. Norwegian Journal of Zoology, 24(524): 419-456.

BRASIL, J., V. L. M. HUSZAR. 2011. O papel dos traços funcionais na ecologia do fitoplâncton continental. Oecologia Australis, 15: 799-834.

BRENDONCK, L, D. C. ROGERS, J. OLESEN, S. WEEKS \& W. R. HOEH. 2008. Global diversity of large branchiopods (Crustacea: Branchiopoda) in freshwater. Hydrobiologia, 595(1): 167-176. DOI: 10.1007/s10750-0079119-9

BRITO, S. L., P. M. MAIA-BARBOSA \& R. P. PINTO-COELHO. 2016. Secondary producti- vity of main microcrustacean species of two tropical reservoirs in Brazil and its relationship with trophic state. Journal of Limnology, 75(2): 320-329. DOI: 10.4081/jlimnol.2016.1267

BURNS, C. W. 1968. The relationship between body size of filter-feeding Cladocera and the maximum size of particle ingested. Limnology and oceanography, 13(4): 675-678. DOI: 10.4319/10.1968.13.4.0675

CARAMUJO, M. J., M.C. CRISPIM \& M. J. BOAVIDA. 1997. Assessment of the importance of fish predation versus copepod predation on life history traits of Daphnia hyalina. Hydrobiologia, 360: 243-252. DOI: 10.1023/ A:1003171828299

CÉRÉGHINO, R., J. BIGGS, B. OERTLI \& S. DE-CLERCK. 2008. The ecology of European ponds: defining the characteristics of a neglected freshwater habitat. Hydrobiologia, 597: 1-6. DOI: 10.1007/s10750-007-9225-8

CETESB. 2000. Zooplâncton de água doce Métodos qualitativo e quantitativo (Norma Técnica L5.304) Companhia Ambiental do Estado de São Paulo. São Paulo, Brazil.

CHAORUANGRIT, L., S. PLODSOMBOON, D. C. ROGERS \& L. SANOAMUANG. 2017. Morphology of mandibles and food size in two fairy shrimps (Branchiopoda: Anostraca) from Thailand. Journal of Crustacean Biology, 1-9. DOI: 10.1093/jcbiol/rux058

CHOUERI, R. B., M. G. G. MELÃO, A. T. LOMBARDI \& A. A. H. VIEIRA. 2007. Effects of cyanobacterium exopolysaccharides on life-history of Ceriodaphnia cornuta SARS. Journal of Plankton Research, 29(4): 339-345. DOI: 10.1093/plankt/fbm020

CIANCIARUSO, M. V., M. A. BATALHA, K. J. GASTON \& O. L. PETCHEY. 2009. Including intraspecific variability in functional diversity. Ecology, 90(1): 81-89. DOI: 10.1890/07-1864.1

COLE, G. A. 1975. Textbook of limnology. The C.V. Mosby Company. Saint Louis. USA.

COLLINSON, N. H., J. BIGGS, A. CORFIELD, M. J. HODSON, D. WALKER, M. WHITFIELD \& P. J. WILLIAMS. 1995. Temporary and permanent ponds: An assessment of the effects of drying out on the conservation value of aquatic macroinvertebrate communities. 
Biological Conservation, 74(2): 125-133. DOI: 10.1016/0006-3207(95)00021-U

CRISPIM, M. C., M. J. BOAVIDA. 2001. Impacto da predação por peixes e copépodes na comunidade zooplanctônica do reservatório do Maranhão (Portugal). Revista Nordestina de Biologia, 15(2): 49-67.

CRISPIM, M. C. \& G. T. D. P.FREITAS. 2005. Seazonal effects on zooplanktonic community in a temporary lagoon of northest Brazil. Acta Limnologica Brasiliensia, 14(4): 385-393.

CROSSETTI, L. O. \& C. E. M. BICUDO. 2005. Structural and functional phytoplankton responses to nutrient impoverishment in mesocosms placed in a shallow eutrophic reservoir (Garças Pond), São Paulo, Brazil. Hydrobiologia, 541:71-85. DOI: 10.1007/ s10750-004-4668-7

DAJOZ, R. 1983. Ecologia Geral. Vozes. Petrópolis. $474 \mathrm{p}$.

DEMOTT, W. R. 1995. The influence of prey hardness on Daphnia's selectivity for large prey. Hydrobiology, 307: 127-138. DOI: 10.1007/978-94-011-0021-2 14

DUMONT, H.J. \& S.V. NEGREA. 2002. Introduction to the Class Branchiopoda. Leiden. Backhuys Publishers. Leiden. 398p.

EDMONDSON, W. T. 1960. Reproductive rates of rotifers in natural populations. Mememorie dell' Istituto Italiano di Idrobiologia, 12: 21-77.

EDMONDSON, W. T. 1965. Reproductive rates of planktonic rotifers as related to food and temperature in nature. Ecological Monographs, 35: 100-111. DOI: 10.2307/1942218

EDMONDSON, W. T. \& G. G. WINBERG. 1971. A manual on methods for the assessment of secondary productivity in fresh waters. Blackwell Scientific Publications (IBP-Handbook, 17), Oxford. UK.

EDMONDSON, W.T. 1974. Secondary production. Verhandlungen des Internationalen Verein Limnologie, 20: 229-272.

EMBRAPA. 2013. Índices Pluviométricos em Minas Gerais (Boletim de Pesquisa e Desenvolvimento) Empresa Brasileira de Pesquisa Agropecuária. Minas Gerais, Brazil.

ESTEVES, F. D. A. 1998. Fundamentos de Limnologia. Rio de Janeiro. Brazil.
FERGUSON, A.J.D., J.M. THOMPSON \& C.S. REYNOLDS. 1982. Structure and dynamics of zooplankton communities maintained in closed systems, with special reference to the algal food supply. Journal of Plankton Research, 4(3): 523-543. DOI: 10.1093/plankt/4.3.523

GLEASON, H. A. 1927. Further Views on the Succession-Concept. Ecology, 8(3): 299-326. DOI: $10.2307 / 1929332$

GOLTERMAN, H. L., R.S. CLYMO, M.A.M. OHNSTAD. 1978. Methods for physical and chemical analysis of freshwaters. 2a ed. Blackwell Scientific Publications (IBP-Handbook, 8), Oxford. USA.

GRAS, R. \& L. SAINT-JEAN. 1969. Biologie des crustacés du Lac Tchad. Cahiers O.R.S.T.O.M., série Hydrobiologie, 3 (3/4): 43-60.

GRAS, R. \& L. SAINT-JEAN. 1976. Durée du développement embryonnaire chez quelques espèces de cladocères et de copépodes du Lac Tchad. Cahiers O.R.S.T.O.M., série Hydrobiologie, 10 (4): 233-254.

GRAS, R. \& L. SAINT-JEAN. 1983. Production du zooplancton du lac Tchad. Revue d'Hydrobiologie Tropicale, 16(1): 57-77.

HARDY, E. \& A. DUNCAN. 1994. Food concentration and temperature effects on life cycle characteristics of tropical Cladocera (Daphnia gessneri Herbst, Diaphanosoma sarci Richard, Moina reticulada (Daday)): I. Development time. Acta Amazonica, 24: 119-134. DOI: 10.1590/1809-43921994242134

HAUSMANN, K., N. HULSMANN \& R. RADEK. 2003. Protistology. Schweizerbart'sche Verlagsbuchhanlung, Stuttgart. 379pp

HERZIG, A. 1983. Comparative studies on the relationship between temperature and duration of embryonic development of rotifers. In: Pejler, B., Starkweather, R., Nogrady, Th. (Eds.). Biology of Rotifers. Proceedings of the Third International Rotifer Symposium held at Uppsala, Sweden, August 30 - September 4, 1982. Hydrobiologia, 104: 237-246. DOI: 10.1007/978-94-009-7287-2_30

HOPP, U. \& G. MAIER. 2005. Implication of the feeding limb morphology for herbivorous feeding in some freshwater cyclopoid copepods. Freshwater Biology, 50: 742-747. DOI: 
10.1111/j.1365-2427.2005.01362.x

HUNTLEY, M. E. \& M. D. G. LOPEZ. 1992. Temperature-Dependent Production of Marine Copepods: A Global Synthesis. The American Naturalist, 140 (2): 201-242. DOI: $10.1086 / 285410$

HUTCHINSON, G.E. 1957. A treatise on limnology. Vol I e II. John Wiley and Sons. NY. USA.

INFANTE, A. \& W. T. EDMONDSON. 1985. Edible phytoplankton and herbivorous zooplankton in Lake Washington. Archiv f'ur Hydrobiologie, 21: 161-171.

IRVINE, K. \& R. WAYA. 1999. Spatial and temporal patterns of zooplankton standing biomass and production in Lake Malawi. Hydrobiologia, 407: 191-205. DOI: 10.1023/ A:1003711306243

KALFF, J. 2002. Limnology. Prentice Hall. NY. USA.

KEPPELER, E. C. 1999. Estudo das populações zooplanctônicas em um lago de meandro abandonado da planície de inundação do Rio Acre (Lago Amapá, Rio Branco-AC, Brasil). M.Sc. Dissertation. Universidade Federal do Acre-UFAC, Rio Branco, Brazil.

KNOECHEL, R. \& L. B. HOLTBY. 1986. Cladoceran filtering rate: body length relationships for bacterial and large algal particles. Limnology and Oceanography, 31(1): 195-199. DOI: 10.4319/1o.1986.31.1.0195

LALIBERTÉ, E. \& P. A LEGENDRE. 2010. Distance-based framework for measuring functional diversity from multiple traits. Ecology, 91(1): 299-305. DOI: 10.1890/08-2244.1

LALIBERTÉ, E., P. LEGENDRE \& B. SHIPLEY. 2014. FD: measuring functional diversity from multiple traits, and other tools for functional ecology. R package version 1.0-12.

LITCHMAN, E., M. D. OHMAN \& T. KIORBOE. 2013. Trait-based approaches to zooplankton communities. Journal of Plankton Research, 35(3): 473-484. DOI 10.1093/plankt/fbt019

LOPES, J. P. 2007. Dinâmica de reprodução e comportamento reprodutivo de branchoneta Dendrocephalus brasiliensis (PESTA, 1921) como incremento na produção de alimento vivo para peixes ornamentais. Dissertation, Universidade Federal do Rio Grande do
Norte, Brazil.

MA, Q., Y. L. XI, J. Y. ZHANG, X. L. WEN \& X. L. XIANG. 2010. Differences in life table demography among eight geographic populations of Brachionus calyciflorus (Rotifera) from China. Limnologica, 40 (2010): 16-22. DOI:10.1016/j.limno.2009.05.002

MACARTHUR, R. H. \& E. O. WILSON. 1967. The theory of island biogeography. Princeton University. Princeton. USA.

MACKERETH, F. Y. H., J. G. HERON \& J. J. TALLING. 1978. Water analysis: some revised methods for limnologists. Freshwater Biology Associate, 36: 120.

MAIA-BARBOSA, P. 2000. Ecologia de cinco espécies de Cladóceros de um lago Amazônico impactado por rejeito de bauxita (Lago Batata, Brasil). PhD Thesis. Universidade Federal do Rio de Janeiro-UFRJ, Rio de Janeiro, Brazil.

MALTCHIK, L., M. A. J. COSTA \& M. D. C. DUARTE. 1999. Inventory of Brazilian semi-arid shallow lakes. Anais da Academia Brasileira de Ciências, 71: 801-808.

MALTCHIK, L. \& E. S. F. MEDEIROS. 2006. Conservation importance of semi-arid streams in north-eastern Brazil: implications of hydrological disturbance and species diversity. Aquatic Conservation: Marine and Freshwater Ecosystems, 16(7): 665-677. DOI: 10.1002/aqc. 805

MARGALEF, R. 1983. Limnologia. Omega. Barcelona, Spain.

MCGILL, B. J., B. J. ENQUIST, E. WEIHER \& M. WESTOBY. 2006. Rebuilding community ecology from functional traits. Trends in Ecology \& Evolution, 21(4): 178-85. DOI: 10.1016/j.tree.2006.02.002

MELÃO, M. G. G. 1997. A comunidade planctônica (fitoplâncton e zooplâncton) e produtividade secundária do zooplâncton de um reservatório oligotrófico. PhD Thesis. Universidade Federal de São Carlos-UFSCAR, São Carlos, Brazil.

MELÃO, M. G. G. 1999. Desenvolvimento e aspectos reprodutivos de cladóceros $e$ copépodes de águas continentais brasileiras. In: M.L.M. POMPÊO (ed.), Perspectivas da limnologia no Brasil. União, São Luís, Brazil. p. 45-57. 
MELÃO, M. G. G. \& O. ROCHA. 2004. Life history, biomass and production of two planktonic cyclopoid copepods in a shallow subtropical reservoir. Journal of Plankton Research, 26: 909-923. DOI: 10.1093/plankt/fbh080

MOREIRA, R.A., O. ROCHA, R. M. SANTOS, E. S. DIAS, F. W. A. MOREIRA \& E. M. ESKINAZI-SANT'ANNA. 2016. Composition, body-size structure and biomass of zooplankton in a high-elevation temporary pond (Minas Gerais, Brazil). Oecologia Australis, 20: 81-93

PAGANO, M. 2008. Feeding of tropical cladocerans (Moina micrura, Diaphanosoma excisum) and rotifer (Brachionus calyciflorus) on natural phytoplankton: effect of phytoplankton size-structure. Journal of Plankton Research, 30(4): 401-414. DOI: 10.1093/plankt/fbn014

PASSOS, R. F. 2012. Influência de Dendrocephalus brasiliensis Pesta, 1921 (Crustacea: Anostraca) na dinâmica da comunidade planctônica em três lagoas temporárias do município de Urucuia, MG. Dissertation. Universidade Federal de São Carlos, São Carlos-SP, Brazil.

PASSOS, R. F. 2017. Estudos ecológicos da comunidade plactônica de vinte e cinco ecossistemas aquáticos temporários tropicais. Ph.D. Thesis. Universidade Federal de São Carlos, São Carlos-SP, Brazil.

PEREZ-BILBAO, A. \& J. GARRIDO. 2009. Evaluacion del estado de conservación de una zona LIC (Gándaras de Budino, Red Natura 2000) usando los coleópteros acuáticos como indicadores. Limnetica, 28(1): 11-22.

PÉREZ-BILBAO, A., C. J. BENETTI \& J. GARRIDO. 2015. Biodiversity and Conservation of Temporary Ponds - Assessment of the Conservation Status of "Veiga de Pontelinares", NW Spain (Natura 2000 Network), Using Freshwater Invertebrates. In: Y. H Lo, J. A. Blanco \& S. Roy (Eds.). Biodiversity in Ecosystems - Linking Structure and Function. DOI: $10.5772 / 59104$

PETCHEY, O. L. \& K. J. GASTON. 2006. Functional diversity: back to basics and looking forward. Ecology Letters, 9(6): 741-58. DOI: 10.1111/j.1461-0248.2006.00924.x

PLA, L., F. CASANOVES \& J. DI RIENZO.
2012. Functional Diversity Indices. In: Quantifying Functional Biodiversity. SpringerBrie$f_{S}$ in Environmental Science, 27-51. DOI 10.1007/978-94-007-2648-2_3

POURRIOT, R. 1977. Food and feeding habits of Rotifera. Arch. Hydrobiol. Beih. Ergebn. Limnol., 8: 243-260.

RABET, N. A. 2006. New species of Brazilian Dendrocephalus (Anostraca, Thamnocephalidae). Zootaxa, 1370: 49-57.

RIETZLER, A.C. 1995. Alimentação, ciclo de vida e análise da coexistência de espécies de na represa de Barra Bonita, São Paulo. Ph.D. Thesis. Universidade de São Paulo - EESC/USP. São Carlos-SP, Brazil.

ROCHA, O., S. SENDACZ, T. MATSUMURATUNDISI. 1995. Composition, Biomass and Productivity of Zooplankton in Natural Lakes and Reservoirs In Brazil. In: José Galizia Tundisi; Carlos Eduardo de Matos Bicudo; Takako Matsumura-Tundisi. (Org.). Limnology in Brazil. 1aed. Academia Brasileira de Ciências, (1aed): 151-166. Rio de Janeiro-RJ. Brazil.

RUTTNER-KOLISKO, A. 1977. Suggestions for biomass calculations of plankton rotifers. Archiv für Hydrobiologie-Beiheft Ergebnisse der Limnologie, 8: 71-76.

SAFI, K., M. V. CIANCIARUSO, R. D. LOYOLA, D. BRITO, M. K. AR-MOUR \& J. A. F. DINIZ-FILHO. 2011. Understanding global patterns of mammalian functional and phylogenetic diversity. Philosophical Transactions of the Royal Society of London B., 366: 2536-2544. DOI: 10.1098/rstb.2011.0024

SAHUQUILLO, M., M. R. MIRACLE, S. M. MORATA \& E. VICENTE. 2012. Nutrient dynamics in water and sediment of Mediterranean ponds across a wide hydroperiod gradient. Limnologica - Ecology and Management of Inland Waters, 42(4): 282-290. DOI: 10.1016/j.limno.2012.08.007

SANTOS, R.M. 2010. Estrutura das comunidades fitoplanctônica e zooplanctônica, com ênfaze na produção secundária do zooplâncton, e fatores ambientais relacionados nos reservatórios do Baixo rio Tietê, SP. M.Sc. Dissertation. Universidade Federal de São Carlos - UFSCar, São Carlos - SP. Brazil. 
SANTOS-WISNIEWSKI, M. J. \& O. ROCHA. 2007. Spatial distribution and secondary production of Copepoda in a tropical reservoir: Barra Bonita, SP, Brazil. Brazilian Journal of Biology, 67: 223-233. DOI: 10.1590/ S1519-69842007000200007

SARMA, S. S. S. \& S. NANDINI. 2002. Studies on functional response and prey selection using zooplankton in the anostracan Chirocephalus diaphanus Prevost, 1803. Hydrobiologia , 486(1): 169-174. DOI: 10.1023/ A:1021398718074

SCHOENBERG, S. A. \& R. E. CARLSON. 1984. Direct and Indirect Effects of Zooplankton Grazing on Phytoplankton in a Hypereutrophic Lake. Oikos, 42(3): 291-302. DOI: $10.2307 / 3544397$

SILVA, L. C. A. 2015. Comunidade zooplanctônica de rios amazônicos na área de influência da Usina Hidrelétrica de Santo Antônio do Madeira, RO: diferentes abordagens no monitoramento. Ph.D. Thesis. Universidade Federal de São Carlos - UFSCar, São Carlos SP, Brazil.

SIMGE. 2015. Relatório de Acompanhamento Trimestral (Relatório de Acompanhamento Climático) Sistema de Meteorologia e Recursos Hídricos de Minas Gerais. Minas Gerais, Brazil.

SMIRNOV, N. N. 1992. The Macrothricidae of the world. The Hague: SPB Academic Publ. $143 \mathrm{p}$.

SMITH, A. B., B. SANDEL, N. J. B. KRAFT \& S. CAREY. 2013. Characterizing scale-dependent community assembly using the functional-diversity-area relationship. Ecology, 94(11): 2392-2402. DOI: 10.1890/12-2109.1

SODRÉ, E. O. 2014. Diversidade funcional da comunidade zooplanctônica em um lago de planicie de inundação. M.Sc. Dissertation. Universidade Federal do Rio de Janeiro UFRJ, Rio de Janeiro - RJ, Brazil.

TUNDISI, J. G. \& T. M. MATSUMURA TUNDISI. 2008. Limnologia. Oficina de Textos. São Paulo-SP. Brazil.

VALDERRAMA, J. C. 1981. The simultaneous analysis of total nitrogen and total phosphorus in natural waters. Marine Chemistry, 10(2): 109-122. DOI: 10.1016/0304-4203
(81)90027-X

VANSCHOENWINKEL, B, J. MERGEAY, T. PINCEEL, A. WATERKEYN, H. VANDEWAERDE, M. SEAMAN \& L. BRENDONCK. 2011 Long distance dispersal of zooplankton endemic to isolated mountaintops-an example of an ecological process operating on an evolutionary time scale. PLoS One, 6(11): e26730. DOI: 10.1371/journal. pone. 0026730

VIEIRA, A. C. B., A. M. A MEDEIROS, L. L. RIBEIRO \& M. C. CRISPIM. 2011. Population dynamics of Moina minuta Hansen (1899), Ceriodaphnia cornuta Sars (1886), and Diaphanosoma spinulosum Herbst (1967) (Crustacea: Branchiopoda) in different nutrients $(\mathrm{N}$ and $\mathrm{P})$ concentration ranges. Acta Limnologica Brasiliensia, 23: 1-9. DOI: 10.4322/actalb.2011.018

VIJVERBERG, J., 1989. Culture techniques for studies on the growth, development and reproduction of copepods and cladocerans under laboratory and in situ conditions: a review. Freshwater Biology, 21: 317-373. DOI: 10.1111/j.1365-2427.1989.tb01369.x

VIOLLE, C., M. L NAYAS, D. VILE, E. KAZAKOU, C. FORTUNEL, I. HUMMEL \& E. GARNIER. 2007. Let the concept of trait be functional! Oikos, 116(5): 882-892. DOI: 10.1111/j.0030-1299.2007.15559.x

WEBB, C. T., J. A. HOETING, G. M. AMES, M. I. PYNE, N. LEROY POFF. 2010. A structured and dynamic framework to advance traits-based theory and prediction in ecology. Ecology Letters, 13(3): 267-83. DOI: 10.1111/j.1461-0248.2010.01444.x

WIGGINS, G. B.; R. J. MACKAY \& I. M. SMITH. 1980. Evolutionary and ecological strategies of animals in annual temporary pools. Archive für Hydrobiologie, 58: 97-206.

WILLIAMS, D. D. 1996. Environmental constraints in temporary fresh waters and their consequences for the insect fauna. Journal of the North American Benthological Society, 15 (4): 634-650. DOI: 10.2307/1467813

WILLIAMS, D. D. 1997. Temporary ponds and their invertebrate communities. Aquatic Conservation: Marine and freschwater ecosystems, 7: 105-117. DOI: 10.1002/(SICI) 
1099-0755(199706)7:2<105::AID-AQC222> 3.0.CO;2-K

WYNGAARD, G. A., C. E. GOULDEN \& A. NOURBAKHSH. 1994. Life history traits of the tropical freshwater copepod Mesocyclops longisetus (Crustacea: Copepoda). Hydrobiologia, 292/293: 423-427. DOI: 10.1007/97894-017-1347-4 53

YÚFERA, M. 1987. Effect of algal diet and temperature on the embryonic development time of the rotifer Brachionus plicatilis in culture. Hydrobiologia, 147: 319-322. DOI: 10.1007/978-94-009-4059-8 43

ZACHARIAS, I., E. DIMITRIOU, A. DEKKER \& E. DORSMAN. 2007. Overview of temporary ponds in the Mediterranean region: Threats, management and conservation issues. Journal of Environmental Biology, 28 (1): 1-9.

Con el apoyo de:

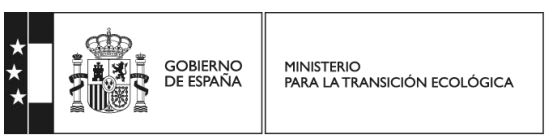

